\title{
Burst occurrence in young massive stellar objects
}

\author{
D. M.-A. Meyer ${ }^{1 \star}$, E. I. Vorobyov ${ }^{2,3}$, V. G. Elbakyan ${ }^{3}$, B. Stecklum ${ }^{4}$, J. Eislöffel ${ }^{4}$ \\ and A. M. Sobolev ${ }^{5}$ \\ ${ }^{1}$ Astrophysics Group, School of Physics and Astronomy, University of Exeter, Exeter EX4 4QL, United Kingdom \\ ${ }^{2}$ Department of Astrophysics, The University of Vienna, Vienna, A-1180, Austria \\ ${ }^{3}$ Research Institute of Physics, Southern Federal University, Stachki 194, Rostov-on-Don, 344090, Russia \\ ${ }^{4}$ Thüringer Landessternwarte Tautenburg, Sternwarte 5, D-07778 Tautenburg, Germany \\ ${ }^{5}$ Astronomical Observatory, Institute for Natural Sciences and Mathematics, Ural Federal University, Ekaterinburg, 620000, Russian Federation
}

Received; accepted

\begin{abstract}
Episodic accretion-driven outbursts are an extreme manifestation of accretion variability. It has been proposed that the development of gravitational instabilities in the proto-circumstellar medium of massive young stellar objects (MYSOs) can lead to such luminous bursts, when clumps of fragmented accretion discs migrate onto the star. We simulate the early evolution of MYSOs formed by the gravitational collapse of rotating $100 \mathrm{M}_{\odot}$ pre-stellar cores and analyze the characteristics of the bursts that episodically accompany their strongly time-variable protostellar lightcurve. We predict that MYSOs spend $\approx 10^{3} \mathrm{yr}(\approx 1.7 \%)$ of their modelled early 60 kyr experiencing eruptive phases, during which the peak luminosity exceeds the quiescent pre-burst values by factors from 2.5 to more than 40. Throughout these short time periods, they can acquire a substential fraction (up to $\approx 50 \%$ ) of their zero-age-main sequence mass. Our findings show that fainter bursts are more common than brighter ones. We discuss our results in the context of the known bursting MYSOs, e.g. NGC6334I-MM1 and S255IR-NIRS3, and propose that these monitored bursts are part of a long-time ongoing series of eruptions, which might, in the future, be followed by other luminous flares.
\end{abstract}

Key words: methods: numerical - stars: flares - stars: massive.

\section{INTRODUCTION}

Massive stars, i.e. stellar objects heavier than $8 \mathrm{M}_{\odot}$, are wellestablished in their preponderant role in the cycle of matter of the interstellar medium of galaxies (Langer 2012). Throughout successive stellar evolutionary phases, from the (post-)main sequence (Meyer et al. 2014) to the supernova phase (Meyer et al. 2015), massive stars are mainly responsible for chemically enriching their surroundings and driving turbulence, which further regulates local star formation. Gravitational interactions and/or massexchanges with close companions modify their internal structures and play an important role in the recent, realistic picture of massive stars evolution (Sana et al. 2012; Schneider et al. 2015). Furthermore, observations of massive young stellar objects (MYSOs, i.e. protostars heavier than $8 \mathrm{M}_{\odot}$ and having a bolometric luminosity $L_{\text {bol }} \geqslant 5 \times 10^{3} \mathrm{~L}_{\odot}$ ) demonstrated that binarity can already exist during the early formation phase of high-mass stellar objects, see also Chini et al. (2012); Mahy et al. (2013); Kraus et al. (2017). Hence, the subsequent main sequence evolution of MYSOs in the Hertzsprung-Russel diagram is a function of mechanisms that are

\footnotetext{
^ E-mail: dmameyer.astro@gmail.com
}

at work already since the initial stage of their formation phase. The evolution of massive stars starts well before the onset of their main sequence phase, at a formation epoch which has been shown to be highly sensitive to mass accretion from the protocircumstellar medium (Hosokawa \& Omukai 2009, Haemmerlé et al. 2016 Haemmerlé \& Peters 2016, Haemmerlé et al. 2017).

Our knowledge of the formation of proto-O stars is mainly driven by observations. Fuente et al. (2001); Testi (2003) and Cesaroni et al. (2006) noticed that massive star formation resembles any other low-mass star formation processes. Indeed their surroundings revealed the presence of converging accretion flows (Keto \& Wood 2006), jets (Caratti o Garatti et al. 2015, Burns et al. 2017; Burns 2018, Purser et al. 2018, Samal et al. 2018), bipolar radiationdriven bubbles filled by ionizing radiation (Cesaroni et al. 2010 . Purser et al. 2016) and collimated pulsed precessing jets (e.g. in Cepheus A, see Cunningham et al. 2009, Reiter et al. 2017). Numerically, global simulations of monolithic gravitational collapse of isolated pre-stellar cores predicted the formation of dense accretion discs (Krumholz et al. 2007), however, such models still clearly suffer from a lack of spatial resolution. Despite advances in supercomputing, it is difficult to fully resolve the central disc, from the outer infalling pre-stellar core material to the stellar surface, including the substructures, such as dense portions of spiral arms and 


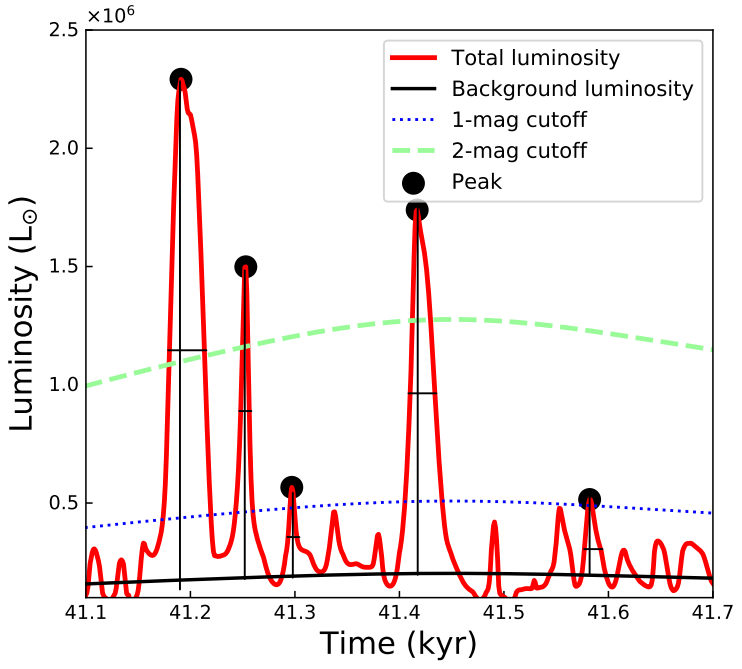

Figure 1. Illustration of the burst filtering method on a series of peaks. On each bursts, their prominence and duration at half maximum are indicated.

eventually dense gaseous clumps developing with the arms. This is mainly due to the fact that their complex thermodynamic structure makes the so-called thin-disc approach less realistic than in the context of, e.g. low-mass star formation, and obliges to tackle the problem with three-dimensional models (Bonnell et al. 1998 Yorke \& Sonnhalter 2002, Peters et al. 2010, Seifried et al. 2011, Harries 2015, Klassen et al. 2016, Harries et al. 2017). Therefore, parameter studies are numerically not conceivable and justify analytic treatments of the problem (Tanaka et al. 2017). Highresolution, self-consistent simulations focusing on the early evolution of MYSOs revealed the existence of accretion-driven outbursts in the context of young massive stars caused by gravitational fragmentation of dense spiral arms followed by migration of gaseous clumps on to the forming star (Meyer et al. 2017). Furthermore, some gaseous clumps can collapse before reaching the star, initiating secondary (low-mass) star formation and forming spectroscopic proto-binaries by direct disc fragmentation (Meyer et al. 2018). These findings orient further investigations on the evolution of massive protostars to their inner proto-circumstellar medium, where mass accretion on to MYSOs occurs.

This is our current picture of the formation of massive stars: theoretical models and available observational data indicate that it is nothing but a scaled-up version of low-mass star formation, retouched by corrections accounting for extreme photospheric conditions via radiation feedback (Richling \& Yorke 1997, Rosen et al. 2017) and its coupling to magnetic fields (Seifried et al. 2011). However, the opaque environment in which they form keeps direct disc observation of deeply-embedded proto-O-typed stars still out of reach of modern instrumentation, although good candidates have been identified (Johnston et al. 2015, Ilee et al. 2016, Forgan et al.2016, Cesaroni et al.2017). A more systematic campaign with ALMA failed in finding evidence of fragmented disc structures within a radius of $1000 \mathrm{au}$ from young protostars (Beuther et al.2017), that were numerically predicted (Meyer et al. 2018).

While a growing number of Keplerian discs around MYSOs is directly detected (Johnston et al. 2015; Ilee et al. 2016, Forgan et al. 2016), some of them including signs of substructures potentially linked to gravitational instability (Ginsburg et al. 2018), the fragmentation scenario proposes an explanation that bridges the gap between strong variability in the lightcurve of MYSOs (an observable) and a precursor mechanism - disc fragmentation at $\sim 100 \mathrm{au}$ from the growing protostar. It is the vivid manifestation of the effectiveness of gravitational non-linear instabilities in the radiatively cooled protostellar surroundings (Gammie 1996) of new-born hot stars, that is regulated by the mechanical heating of dense spiral arms (Rogers \& Wadsley 2012). Accretion bursts caused by disk fragmentation followed by inward migration of dense clumps onto the star have the advantage to be well-understood in the context of young low-mass stars (Vorobyov \& Basu 2005, 2015, Machida \& Matsumoto 2011, Zhao et al. 2018, Nayakshin \& Lodato 2012). In the high-mass context, bursts constitute a formidable insight into the recent past of the circumprotostellar medium, allowing us to probe the not-yet directly observable inner disc regions. Coincidently, such disc-mediated bursts have recently been observed, e.g. from the MYSOs S255IR NIRS 3 (Fujisawa et al. 2015, Stecklum et al. 2016, Caratti o Garatti et al. 2017) or from NGC6334IMM1 (Hunter et al. 2017).

This work aims at studying the properties and occurrence of accretion-driven outbursts from massive stars. We perform threedimensional gravito-radiation-hydrodynamical simulations of collapsing $100 \mathrm{M}_{\odot}$ rotating pre-stellar cores to model the longterm evolution of gravitationally-unstable accretion discs around MYSOs. We extract from those simulations both the highlyvariable accretion rate histories and luminosity curves interspersed with episodic bursts. The bursts are generated by the accretion of dense material from spiral arms in the disk and/or by the inward migration of fragments as described in Meyer et al. (2018). This picture is well-supported by observations of the spiral filament that is feeding the candidate disc around the young massive stellar object MM1-Main (Maud et al.2017), by the observation of a young stellar object fed by a gaseous clump in the double-cored system G350.69-0.49 (Chen et al. 2017). We separate the bursts of our modelled MYSOs using the method developed in the context of FU-Orionis bursts (Vorobyov \& Basu 2015, Vorobyov et al. 2018). The intensity and duration of individual bursts are analysed and compared with each other. We evaluate their occurrence during the early pre-main sequence phase of stellar evolution and estimate the amount of time our MYSOs spend experiencing accretion bursts.

This study is organized as follows. In Section 2, we review the methods and initial conditions that we used to perform numerical hydrodynamical simulations of the circumstellar medium of our massive protostars. The obtained total internal luminosities of young massive protostars, exhibiting violent luminous spikes, are analysed in order to study the burst occurence throughout their early pre-main sequence star's live. The accretion rate evolution onto the MYSOs, the analysis of their luminosity histories and their occurence are presented in Section 3, and we investigate the effects of stellar inertia onto the burst activity in Section 4. In Section 5, we detail the different types of accretion bursts happening throughout the early formation phase of MYSOs, investigate the effects of the burst phenomenon in the growth of young massive protostars, further discuss our results in the context of observations. Finally, we conclude in Section 6.

\section{METHODS}

In this section, we provide a concise description of the gravitoradiation-hydrodynamical simulations utilised to derive the mass transport rate onto our forming high-mass stars and we briefly sum- 

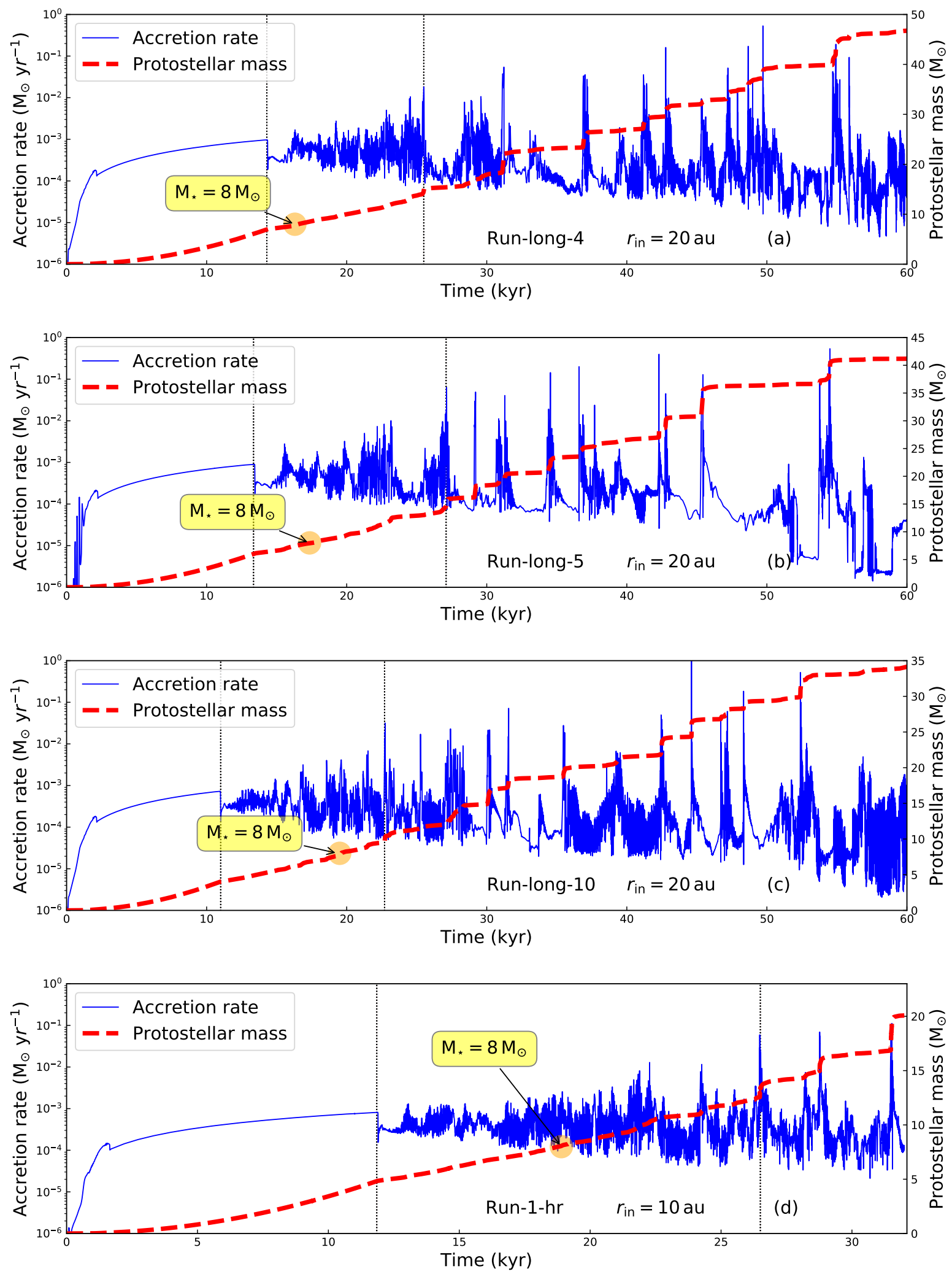

Figure 2. Mass accretion rate (thin solid line, in $\mathrm{M}_{\odot} \mathrm{yr}^{-1}$ ) and protostellar mass evolution (thick dashed line, in $\mathrm{M}_{\odot}$ ) in our simulations of $100 \mathrm{M}_{\odot}$ solid-body-rotating pre-stellar cores with $\beta=4 \%$ (a), $5 \%$ (b), $10 \%$ (c) and Run1-hr (d). 
Table 1. Initial characteristics of our $100 \mathrm{M}_{\odot}$ solid-body-rotating prestellar cores. The table gives the inner sink cell radius $r_{\text {in }}$, the ratio of rotational-to-gravitational energy $\beta$, final simulation time $t_{\text {end }}$ and indicates if the stellar inertia is included in the models.

\begin{tabular}{|c|c|c|c|c|}
\hline Models & $r_{\text {in }}(\mathrm{au})$ & $\beta(\%)$ & $t_{\text {end }}(\mathrm{kyr})$ & Wobbling \\
\hline Run - long $-4^{(a)}$ & 20 & 4 & 60 & no \\
\hline Run - long $-5^{(a)}$ & 20 & 5 & 60 & no \\
\hline Run - long $-10^{(a)}$ & 20 & 10 & 60 & no \\
\hline Run1 - hr ${ }^{(b)}$ & 10 & 4 & 32 & no \\
\hline Run - without $^{(a)}$ & 12 & 4 & 40 & no \\
\hline Run $-\operatorname{with}^{(a)}$ & 12 & 4 & 40 & yes \\
\hline
\end{tabular}

(a) This work, (b) Meyer et al. (2018,

marise the subsequent analysis method of their total luminosity history.

\subsection{Numerical model}

The numerical simulations are performed using a midplanesymmetric computational domain, that we initialise with a $100 \mathrm{M}_{\odot}$ solid-body-rotating pre-stellar core of uniform temperature $T_{\mathrm{c}}=$ $10 \mathrm{~K}$ and of an initially spherically symmetric density distribution profiled as $\rho(r) \propto r^{\beta_{\rho}}$, with $\beta_{\rho}=-3 / 2$ and $r$ the radial coordinate. Its inner radius $r_{\text {in }}$ constitutes a semi-permeable sink cell fixed onto the origin of the domain and its outer radius, assigned to outflow boundary conditions, is located at $R_{\mathrm{c}}=0.1 \mathrm{pc}$. The grid mesh maps the domain $\left[r_{\mathrm{in}}, R_{\mathrm{c}}\right] \times[0, \pi / 2] \times[0,2 \pi]$ made of $N_{\mathrm{r}}=138 \times N_{\theta}=21 \times N_{\phi}=138$ grid zones minimum. It expands logarithmically along the radial direction $r$, goes as a cosine in the polar direction $\theta$ and is uniformly spaced along the azimuthal direction $\phi$. Such grid intrinsically saves computing resources while high-spatially resolving the inner region of the midplane with a reduced total number of grid zones. We follow the gravitational collapse of the pre-stellar core and the early formation and evolution of the subsequent circumstellar accretion disc that surrounds the protostar. We calculate the material loss $\dot{M}$ through the sink cell as directly being the accretion rate onto the protostar, whose properties (e.g., stellar radius and photospheric luminosity) are time-dependently updated using the pre-calculated protostellar evolutionary tracks of Hosokawa \& Omukai (2009). Hence, our estimate of the protostellar radius are in accordance with the calculations of Hosokawa \& Omukai (2009), as in Meyer et al. (2017) 2018). The different models are therefore characterised by the radius of the sink-cell $r_{\text {in }}$ and the spin of the pre-stellar core that is parametrised by its ratio of rotational-by-gravitational energy $\beta$. Two additional simulations are performed in order to test the validity of our assumption consisting in neglecting the stellar inertia (Regály \& Vorobyov 2017; Hirano et al. 2017). The comparison is effectuated in our Section 4. We use a slightly larger sink-cell radius $\left(r_{\text {in }}=20 \mathrm{au}\right)$ than in our pilot study (see Meyer et al.2017), which allows us to reach longer integration times $t_{\text {end }}$ without dealing with dramatic time-step restrictions in the innermost grid zones. Such models, whose characteristics are summarised in Tab. 1 permit to obtain a large burst population, which is more suitable for our study.

We solve the equations of gravito-radiation-hydrodynamics with the PLUTO code ${ }^{1}$ (Mignone et al. 2007 2012). Direct proto-

\footnotetext{
1 http://plutocode.ph.unito.it/
}

stellar irradiation feedback and radiation transport in the accretion disc are taken into account within the gray approximation with the scheme of Kolb et al. (2013 ${ }^{2}$ adapted in the fashion of Meyer et al. (2018). This two-fold algorithm ray-traces photon packages from the protostellar surface and flux-limited diffuses their propagation into the disc. Although other sophisticated methods have been developed for non-uniform Cartesian coordinate systems (Klassen et al. 2016, Rosen et al. 2017), it allows us to accurately treat both the inner heating and the outer cooling of our irradiated discs (Vaidya et al. 2011). Equivalent radiation-hydrodynamics methods have also been presented in e.g. Commerçon et al. (2011), Flock et al. (2013) and Bitsch et al. (2014). The opacity description as well as the estimate of the local dust properties are similar as in Meyer et al. (2018), where gas and dust temperature are calculated assuming the equilibrium between the silicate grains temperature and the total radiation field. We model the stellar gravity by calculating the total gravitational potential of the central protostar and include the self-gravity of the gas 3 with the method of Black \& Bodenheimer (1975) by solving the Poisson equation using the PETSC library ${ }^{4}$ As in the other papers of this series, we neglect turbulent viscosity by assuming that the most efficient angular momentum transport mechanism are the gravitational torques generated once a self-gravitating disc has formed after the collapse (see also Hosokawa et al. 2016). We refer the reader interested in further reading about the numerical method to Meyer et al. (2018).

\subsection{Internal luminosities of accreting protostars}

The internal luminosity history of our accreting young stars is calculated as the sum of their photospheric luminosity $L_{\star}$, interpolated from the protostellar tracks of Hosokawa \& Omukai (2009), plus the accretion luminosity $L_{\text {acc }}=G M_{\star} M / 2 R_{\star}$, where $G$ is the universal gravitational constant, $M_{\star}$ is the stellar mass, $\dot{M}$ is the accretion rate onto the protostar and $R_{\star}$ is the protostellar radius, respectively. Using pre-calculated tracks is a good compromise between the direct (but computationally-costly) coupling of the gravito-radiation-hydrodynamics simulations to a stellar evolution code, which updates in real time the stellar properties by solving the stellar structure and the simple treatment of $L_{\star}$ as a function of the stellar effective temperature. We analyse the total luminosity histories using four models, namely runs Run-long-4\%, Runlong-5\% and Run-long-10\%, plus the model Run1-hr of Meyer et al. (2018). While Run-long-4\%, Run-long-5\% and Run-long$10 \%$ form a homogeneous ensemble of simulations that assume similar grid resolution and sink-cell radius, model Run1-hr differs by its smaller sink-cell radius and higher grid resolution (see Tab. 1. The main limitation of such simulations, in addition to spatial resolution, is the radius of the inner sink cell, which should be as small as possible. The maximum radius of our sink cell is 20 $\mathrm{au}$, which is smaller than in some other studies (up to $50 \mathrm{au}$, see in Hosokawa et al. 2016). Smaller sinks are very computationally expensive due to strong limitations imposed by the Courant timestep condition. We note that the numerical convergence of runs with a grid resolution as ours was demonstrated in Section 5 of Meyer et al. (2018). Our overall goal consists in identifying the accretion bursts, their numbers, characteristics and occurrence, in order to

\footnotetext{
2 http://www.tat.physik.uni-tuebingen.de/ pluto/pluto_radiation/

3 https://shirano.as.utexas.edu/SV.html

4 https://www.mcs.anl.gov/petsc/
} 

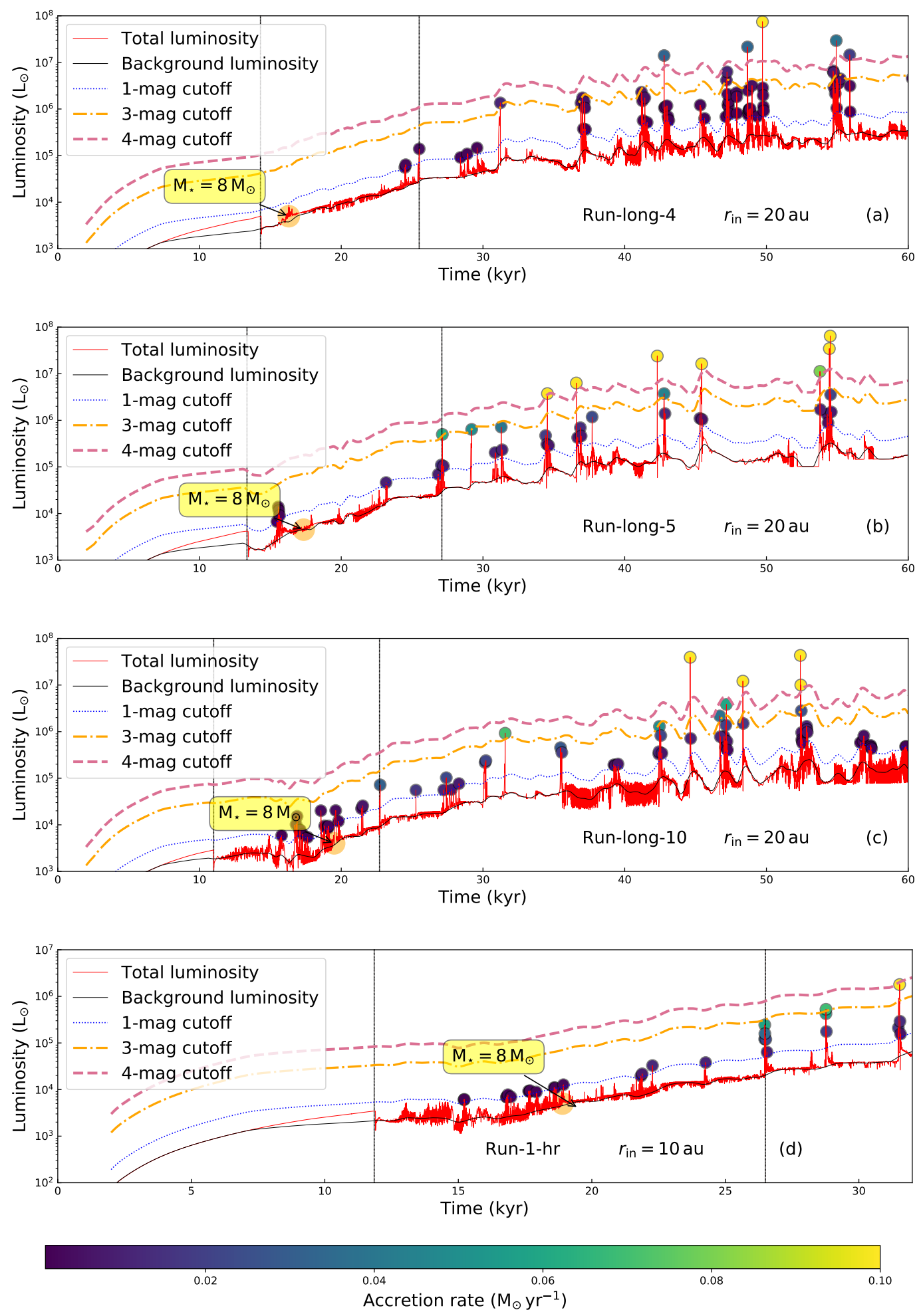

Figure 3. Total luminosity (thin solid red line, in $\mathrm{L} \odot$ ) as a function of time (in kyr) of our models with $\beta=4 \%$ (a), $5 \%$ (b), $10 \%$ (c) and Run1-hr (d). The panels show the background luminosity (thin solid black line) and the dotted lines indicate the luminosity greater than $2.5, \approx 6.3$ and $\approx 39.0$ times the background luminosity (1,2- and 4-mag cutoff), respectively. The color bar indicates the accretion rate for the filled circles. 
Table 2. Summary of burst characteristics. $N_{\mathrm{bst}}$ is the number of bursts at a given magnitude cut-off. $L_{\max } / L_{\min } / L_{\operatorname{mean}}$ are the maximum, minimum and mean burst luminosities, respectively. Similarly, $\dot{M}_{\max } / \dot{M}_{\text {min }} / \dot{M}_{\text {mean }}$ are the maximum, minimum and mean accretion rates through the central sink cell and $t_{\mathrm{bst}}^{\max } / t_{\mathrm{bst}}^{\min } / t_{\mathrm{bst}}^{\operatorname{mean}}$ are the maximum, minimum and mean bursts duration, while $t_{\mathrm{bst}}^{\text {tot }}$ is the integrated bursts duration time throughout the star's live.

\begin{tabular}{|c|c|c|c|c|c|}
\hline Model & $N_{\text {bst }}$ & $L_{\max } / L_{\min } / L_{\text {mean }}\left(10^{5} \mathrm{~L}_{\odot} \mathrm{yr}^{-1}\right)$ & $\dot{M}_{\max } / \dot{M}_{\min } / \dot{M}_{\text {mean }}\left(\mathrm{M}_{\odot} \mathrm{yr}^{-1}\right)$ & $t_{\mathrm{bst}}^{\max } / t_{\mathrm{bst}}^{\min } / t_{\mathrm{bst}}^{\operatorname{mean}}(\mathrm{yr})$ & $t_{\mathrm{bst}}^{\mathrm{tot}}(\mathrm{yr})$ \\
\hline \multicolumn{6}{|c|}{ 1-mag cutoff } \\
\hline Run-long-4\% & 34 & $14.7 / 0.56 / 7.39$ & $0.018 / 0.003 / 0.007$ & $39 / 5 / 14$ & 471 \\
\hline Run-long-5\% & 21 & $15.1 / 0.067 / 4.4$ & $0.019 / 0.001 / 0.008$ & $88 / 9 / 26$ & 553 \\
\hline Run-long-10\% & 49 & $13.1 / 0.054 / 3.78$ & $0.023 / 5.13 \times 10^{-4} / 0.006$ & $94 / 5 / 16$ & 790 \\
\hline Run-hr & 24 & $2.9 / 0.06 / 0.54$ & $0.029 / 0.001 / 0.008$ & $100 / 5 / 51$ & 1231 \\
\hline Total all models & 128 & $15.1 / 0.054 / 4.03$ & $0.029 / 5.13 \times 10^{-4} / \mathbf{0 . 0 0 7}$ & $100 / 5 / 27$ & 761 \\
\hline Run-without & 9 & $4.31 / 0.11 / 1.49$ & 0.0177 / $0.0022 / 0.0081$ & $107 / 9 / 38$ & 340 \\
\hline Run-with & 6 & $3.60 / 0.123 / 1.19$ & $0.0262 / 0.0024 / 0.0131$ & $66 / 9 / 29$ & 173 \\
\hline \multicolumn{6}{|c|}{ 2-mag cutoff } \\
\hline Run-long-4\% & 22 & $45.6 / 10.5 / 25.7$ & $0.036 / 0.012 / 0.022$ & $55 / 3 / 13$ & 285 \\
\hline Run-long-5\% & 6 & $35.7 / 6.4 / 15.3$ & $0.049 / 0.010 / 0.027$ & $17 / 6 / 12$ & 69 \\
\hline Run-long-10\% & 13 & $28 / 0.15 / 7.65$ & $0.049 / 0.003 / 0.021$ & $74 / 6 / 20$ & 264 \\
\hline Run-hr & 3 & $4.26 / 1.59 / 2.76$ & $0.059 / 0.035 / 0.050$ & $61 / 30 / 44$ & 131 \\
\hline Total all models & 44 & $45.6 / 0.15 / 12.9$ & $0.059 / 0.003 / 0.03$ & $74 / 3 / 22$ & 187 \\
\hline & & & $00170+00041,0024$ & & \\
\hline Run-without & 5 & $8.05 / 1.90 / 4.64$ & $0.0479 / 0.0241 / 0.0343$ & $34 / 5 / 18$ & 88 \\
\hline Run-with & 5 & $9.80 / 1.57 / 4.12$ & 0.0567 / $0.0175 / 0.0397$ & $33 / 7 / 21$ & 105 \\
\hline \multicolumn{6}{|c|}{ 3-mag cutoff } \\
\hline Run-long-4\% & 5 & $65.7 / 13.5 / 48.8$ & $0.054 / 0.035 / 0.044$ & $29 / 4 / 12$ & 60 \\
\hline Run-long-5\% & 2 & $37.4 / 4.99 / 21.2$ & $0.062 / 0.045 / 0.053$ & $35 / 8 / 22$ & 43 \\
\hline Run-long-10\% & 3 & $38.5 / 9.24 / 23.2$ & $0.071 / 0.038 / 0.056$ & $8 / 3 / 6$ & 17 \\
\hline Run-hr & 2 & $17.9 / 5.28 / 11.6$ & $0.111 / 0.069 / 0.090$ & $51 / 50 / 51$ & 101 \\
\hline Total all models & 12 & $65.7 / 4.99 / 26.2$ & $0.111 / 0.035 / 0.061$ & $51 / 3 / 23$ & 55 \\
\hline Run-without & 2 & $12.2 / 8.55 / 10.4$ & $0.105 / 0.083 / 0.094$ & $6 / 4 / 5$ & 10 \\
\hline Run-with & 2 & $17.8 / 16.2 / 17.0$ & $0.121 / 0.059 / 0.0895$ & $11 / 7 / 9$ & 18 \\
\hline \multicolumn{6}{|c|}{ 4-mag cutoff } \\
\hline Run-long- $4 \%$ & 5 & $745 / 140.5 / 308$ & $0.524 / 0.093 / 0.226$ & $9 / 2 / 5$ & 27 \\
\hline Run-long-5\% & 8 & $644.3 / 37.5 / 221.3$ & $0.438 / 0.080 / 0.215$ & $10 / 2 / 4$ & 32 \\
\hline Run-long-10\% & 4 & $432.4 / 100 / 261.9$ & $0.925 / 0.101 / 0.424$ & $7 / 2 / 4$ & 14 \\
\hline Run-hr & - & - & - & - & - \\
\hline Total all models & 17 & $745 / 37.5 / 263.7$ & $0.925 / 0.080 / 0.288$ & $10 / 2 / 4$ & 24 \\
\hline & & & - & & \\
\hline Run-without & - & - & - & - & - \\
\hline Run-with & 1 & $51.2 / 51.2 / 51.2$ & $0.16 / 0.16 / 0.16$ & $4 / 4 / 4$ & 4 \\
\hline
\end{tabular}

determine the time that young massive stars spend bursting during their early formation phase, and the mass they accrete while experiencing those luminous events.

We use the statistical method developed in the context of accreting low-mass protostars in Vorobyov \& Basu (2015); Vorobyov et al. (2018). It aims at discriminating between the background secular variability accounting for anisotropies in the accretion flow generated by the presence of enrolled spiral arms, and the various luminosity bursts generated by the accretion of dense gaseous clumps. It can be summarised as follows. A background luminosity $L_{\mathrm{bg}}$ is calculated by filtering out all accretion-driven events such that a smooth function of time is obtained. The quantity $L_{\mathrm{bg}}$ is taken as being $L_{\mathrm{acc}}+L_{\star}$ if the instantaneous accretion rate $\dot{M}(t)$ is smaller than $\dot{M}_{\text {crit }}=5 \times 10^{-4} \mathrm{M}_{\odot} \mathrm{yr}^{-1}$ and as $L_{\text {acc }} \times \dot{M}_{\text {crit }} / \dot{M}(t)+L_{\star}$ otherwise, which has the effect to filter out all strong accretion bursts from the stellar internal luminosity. We then derive the intensities and durations of so-called 1-mag, 2mag, 3-mag, and 4-mag accretion-driven outbursts. In particular, by the 1-mag outburst we mean all outbursts with luminosity greater than 2.5 times the background luminosity (1-mag cutoff), but is lower than $2.5^{2}$ times the background luminosity (2-mag cutoff).
The 2-, 3-, and 4-mag bursts are defined accordingly, with an exception of the 4-mag bursts having no upper limit (Table 2). The filtering method additionally insures that (i) the accretion-driven bursts duration is short enough so that slow increases and decreases of the total internal stellar luminosity are not mistaken as outbursts and that (ii) low-intensity kinks of less than 1-mag present in the lightcurves are also not interpreted as accretion outbursts, because they may be produced by boundary effects. Fig. 1 illustrates the burst filtering method on a series of successive accretion peaks on which their prominence (luminous intensity with respect to $L_{\mathrm{bg}}$ ) and duration (width at half-maximal prominence) are plotted. Statistical quantities such as variance and standard deviations of the collection of selected bursts are finally derived (Table 3). We refer the reader interested in further details about the bursts analysis method to Vorobyov \& Basu (2015) and Vorobyov et al. (2018).

\section{EPISODIC ACCRETION-DRIVEN BURSTS}

In this section, we present the accretion rate histories of our set of simulations and we describe their corresponding protostellar lu- 

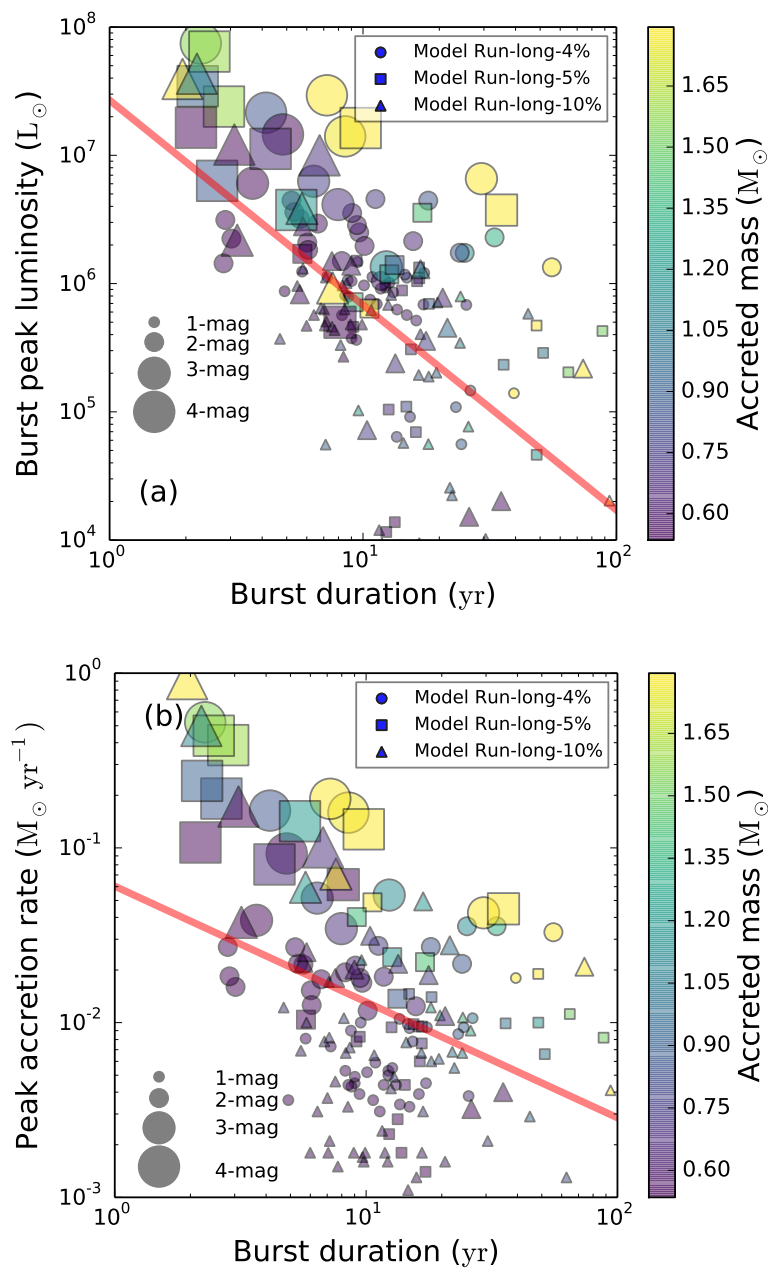

Figure 4. Correlation between burst duration versus peak luminosity (a) and burst duration versus peak accretion rate (b) for each individual bursts, with colours representing the mass accreted by the protostar during the bursts.

minosities. Then, we analyse the burst characteristics in order to extract their properties such as their duration and occurence.

\subsection{Accretion rate histories}

Fig 2 shows the accretion rate evolution (in $\mathrm{M}_{\odot} \mathrm{yr}^{-1}$ ) onto our protostars formed out of pre-stellar cores with initial rotational-togravitational energy ratios $\beta=4 \%$ (Run-long-4, panel a), $5 \%$ (Runlong-5, panel b), 10\% (Run-long-10, panel c) and Run1-hr (panel d). The effects of accretion on the protostar's growth is explicitly illustrated in the figure by plotting the evolution of the protostellar mass (thick dotted red line, in $\mathrm{M}_{\odot}$ ) over the accretion rate histories (thin solid blue lines). Additionally, an orange dot marks the time instance when the young star becomes a massive object $\left(\mathrm{M}_{\star} \geqslant 8 \mathrm{M}_{\odot}\right)$. The dotted black vertical lines separate different sequences of the young star's life characterised by distinct accretion regimes. The first vertical line corresponds to the onset of disc formation, when the free-fall of the envelope material onto the star ceases and the star starts gaining its mass via accretion from the circumstellar disc. The second vertical line marks the beginning of the first sudden increase of the accretion rate that is associated to $a \geqslant 2$-mag burst (see Section 3.2. These two vertical lines high- light distinct protostellar mass accretion regimes corresponding to several different accretion phases.

First, the collapse of the parent pre-stellar core generates an infall of material through the sink cell, producing the initial increase of the mass flux through the inner boundary. During the initial $\approx 2 \mathrm{Myr}$, the mass accretion rate increases from $\approx 10^{-6}$ to $\approx 10^{-4} \mathrm{M}_{\odot} \mathrm{yr}^{-1}$ and a further increase of the collapsing gas radial velocity happens over the next $\approx 10 \mathrm{kyr}$, increasing $\dot{M}$ from $\approx 10^{-4}$ to slightly less than $\approx 10^{-3} \mathrm{M}_{\odot} \mathrm{yr}^{-1}$. The latter value is the typical rate at which MYSOs are predicted to accrete (Hosokawa \& Omukai 2009). The time instance of disc formation is a function of the initial spin of the pre-stellar core, see also Meyer et al. (2018). When the disc forms (first vertical line of Figs. 2 a-d), moderate variability appears immediately in the accretion flow as a result of anisotropies in the density and velocity fields close to the MYSOs. The values of $\dot{M}(t)$ evolve such that the variation amplitude gradually augments up to spanning over almost 2 orders of magnitudes, from $\approx 10^{-4}$ to $\approx 10^{-2} \mathrm{M}_{\odot} \mathrm{yr}^{-1}$ (Fig.2p). A slight change in the slope of the mass evolution is associated with the beginning of this second, variable-accretion phase. All our protostars become heavier that $8 \mathrm{M}_{\odot}$ and therefore enter the massive regime of stellar masses in this phase (see orange dot). Although the increase of the stellar mass is still smooth (thick dotted red line), step-like augmentations due to the fast accretion of dense material by the sink cell happen, e.g. at times $\approx 23 \mathrm{kyr}$ in model Run-long-5 and $\approx 24$ kyr in model Run-1-hr (thick red dotted line of Fig. 2p,d), when the protostellar mass has reached $\approx 10 \mathrm{M}_{\odot}$. Those accretion spikes grow in number and intensity as a function of time, because the discs surrounding the MYSOs, while growing in size, develop complex morphologies by adopting the filamentary structure of self-gravitational rotating discs. A more detailed description of the early disc structure evolution of massive irradiated self-gravitating discs can be found in Meyer et al. (2018).

The variations amplitude in the accretion rate continues to increase during about $10-15 \mathrm{kyr}$ after disc formation, until the MYSOs enter the next evolution phase marked by the second dotted vertical line. For each model, such events are highlighted in Fig. 2 with the second vertical line. Without stopping the baseline accretion (at the average rate of $\approx 10^{-3} \mathrm{M}_{\odot} \mathrm{yr}^{-1}$ ), this evolution phase is regularly interspersed with strong accretion spikes which generate luminosity outbursts via the mechanism highlighted in Meyer et al. (2017). The essence of this mechanism is gravitational fragmentation of discs spiral arms, which extend up to radii $\approx 100-1000 \mathrm{au}$, followed by inward migration and infall of gaseous clumps on the protostar. At this stage, the mass of the protostar grows essentially due to those strong accretion events and the mass gained by accretion during the quiescent phase is negligible (see also discussion in Section 5.3). The difference in the spike occurrence is only a function of the initial gravitational-to-kinetic energy $\beta$ (our Table 1), as it is the sole parameter by which models Run-long-4, Run-long-5 and Run-long-10 differ (Fig. 2a-c). At $t_{\mathrm{end}}=60 \mathrm{kyr}$, the MYSOs have masses of about $\approx 47, \approx 42$ and $\approx 34 \mathrm{M}_{\odot}$, respectively. Their growth should continue up to the exhaustion of disc's envelope mass reservoir. When the envelope no more fuels the star-disc system, both infall rate onto the disc and accretion rate onto the protostar should gradually decrease and stop (not modeled in our simulations) as it happens in the context of low-mass star formation (Vorobyov 2010, Vorobyov et al. 2017). 


\subsection{Protostellar luminosities}

Fig 3 plots the total, bolometric luminosity of the MYSOs evolution (photospheric plus accretion luminosity, in $\mathrm{L}_{\odot}$ ) as a function of time (in kyr) of our protostars generated by the collapse of prestellar cores of initial kinetic-by-gravitational energy ratio $\beta=4 \%$ (Run-long-4, panel a), 5\% (Run-long-5, panel b), 10\% (Run-long10, panel c) and Run1-hr (panel d). The thin dotted red line represents the total luminosity, the black solid line corresponds to the background luminosity $L_{\mathrm{bg}}$, while the dotted thin blue line, dashed dotted orange line and dashed violet lines show the limit of the 1-, 3 - and 4-magnitudes cutoff with respect to $L_{\mathrm{bg}}$. The overplotted dots are the peak accretion rates for the burst which peak luminosity is at least 2.5 times brighter than their quiescent pre-flare protostellar luminosity. Shown in the $0.01-0.1 \mathrm{M}_{\odot}$ range, their colour corresponds to the peak's accretion rate (in $\mathrm{M}_{\odot} \mathrm{yr}^{-1}$ ). As in Fig 2 the orange dot indicates the time instance when the star becomes a massive object $\left(M_{\star} \geqslant 8 \mathrm{M}_{\odot}\right)$ and the dotted black vertical lines separate the above described mass regimes: the onset of disc formation, and the beginning of the above described spiked accretion phase.

The initial free-fall gravitational collapse of the pre-stellar cores corresponds to a luminosity phase $<10^{3} \mathrm{~L}_{\odot}$ which last $\approx 10$ kyr. As demonstrated in Fig. 7 of Meyer et al. (2018), the protostars still have a negligible accretion luminosity: because of still relatively small stellar mass and/or large stellar radius, their luminosity is governed by their photospheric component $L_{\mathrm{bol}} \approx$ $L_{\star} \leqslant 10^{4} \mathrm{~L}_{\odot}$ (Figs. 3 a-d). Stellar objects in such phase are likely not observable, as not bright enough to produce photons able to escape their opaque embedded host cores. Once the circumstellar disc forms (first black vertical line), the luminosity adopts a timedependent behaviour which reflects the protostellar accretion rate history (Figs. 2a). All our models exhibit a spectrum of burst magnitudes from 1-mag bursts to 4-mag bursts, except for model Run1-hr, which lacks the 4-mag bursts (perhaps due to shorter simulation times, $\approx 35 \mathrm{kyr}$, as compared to other models). However, the total number of the bursts and the number of the burst of a certain magnitude vary from model to model.

Indeed, a finer grid resolution resolves better the Jeans length. That, in its turn, allows us to treat more realistically the disc gravitational instabilities and reveal smaller disc fragments, a subset of which of will end up migrating onto the protostar and generating (milder intense) bursts. As an example, our model Run-long-4 has a single 1-mag burst (Fig. 33), whereas our model Run-1-hr exhibits 9 of them over roughly the same time interval (between the two vertical black lines, see Fig. 3d). A more thorough discussion of the resolution-dependence of numerical investigations on disc fragmentation can be found in Meyer et al. (2018). Similarly, bursts are more frequent at higher $\beta$-ratio, e.g. Run-long- 4 has a single 1mag burst but Run-long-10 has more than a dozen (Fig. 3 a,c). This illustrates the effects of the initial conditions on the disc dynamics. We also notice that, in the case of our fast-rotating models, bursts develop earlier in time, e.g. models Run-long-10 and Run-long-4 have their first 1 -mag burst at a time $\approx 15 \mathrm{kyr}$ and $\approx 26 \mathrm{kyr}$, respectively.

After the first strong ( $\geqslant 2$-mag) burst, the variations of the total protostellar luminosity gradually increases as the accretion luminosity overwhelms the photospheric contribution to the total luminosity. This is tied to the rising complexity of the disc pattern: the multiple spiral arms and gaseous clumps forming in the disc experience a chaotic motion and violently interact by formation, destruction, merging and migration in the star's surroundings (Meyer et al.
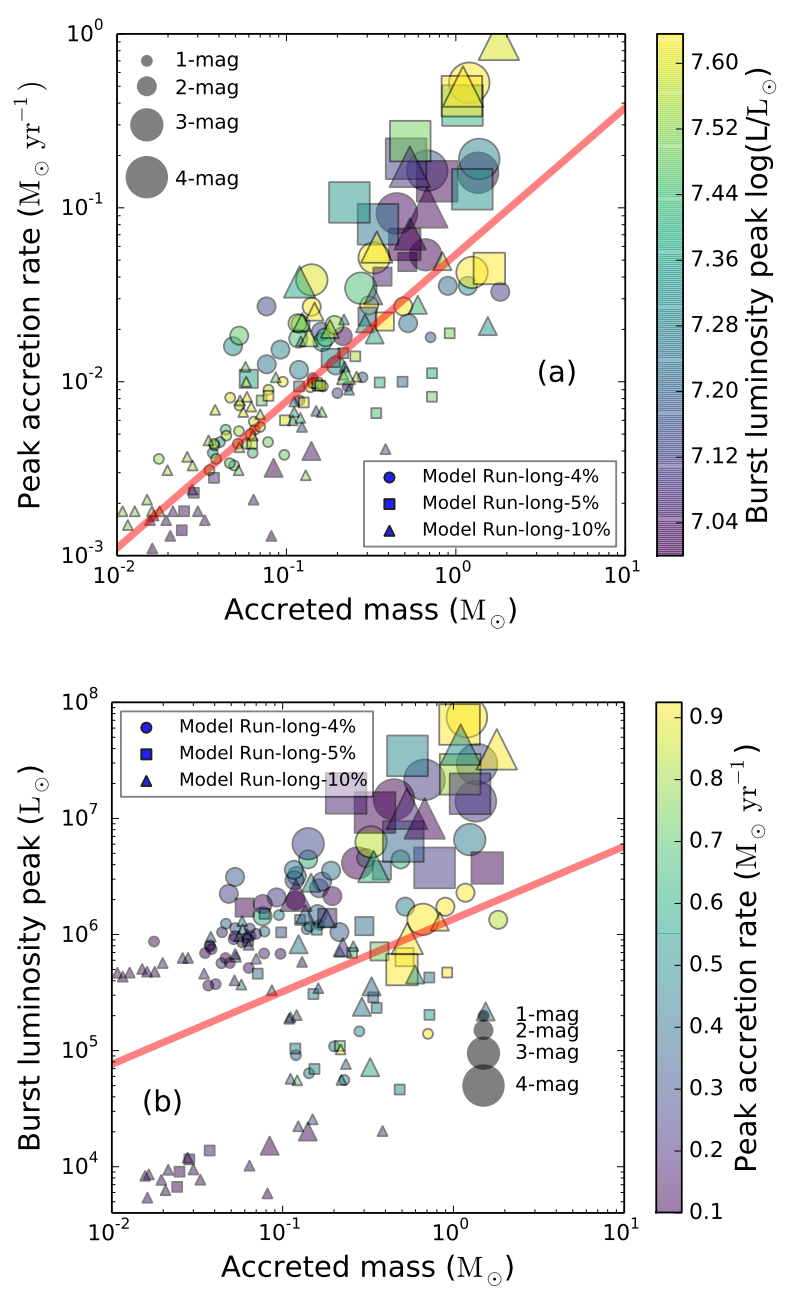

Figure 5. Correlation between accreted mass versus peak accretion rate (a) and accreted mass versus peak luminosity (b) for each individual burst, with colours representing the peak luminosity (a) and the peak accretion rate (b), respectively.

2018). The number of bursts per unit time consequently increases after $\approx 30 \mathrm{kyr}$ and the protostellar lightcurve is shaped as a forest of accretion-driven peaks. The burst intensity is not a monotonic function of time as high-intensity bursts (e.g., 3- and 4-mag) can be interspersed by lower-intensity ones (e.g., 1- and 2-mag) or by quiescent burstless phases. Bursts often appear grouped as a collection of different magnitude bursts on a rather small time interval, see e.g. our model Run-long-4 at times $45-50 \mathrm{kyr}$.

Note that accretion-driven bursts can appear either isolated or clustered. Single bursts are provoked by the infall of a gaseous clump which is detached from its parent spiral arm and experiences a migration towards the central MYSO, see mechanism described in Meyer et al. (2017). They correspond to the accretion of relatively large amount of compact circumstellar material over rather short timescales ( $\sim 10 \mathrm{yr}$ ), which produces a unique highluminosity burst. The rapid successive accretion of several migrating clumps from different parent arms, the merging of clumps into inhomogeneous gaseous structures or even the migration of clumps that separate an inner portion of spiral arms into two segment make the accretion pattern more complex and can produce different a type of lightcurves exhibiting lower-intensity, clustered bursts, in a 
manner consistent with the description of Vorobyov \& Basu (2015) in low-mass star formation.

\subsection{Bursts characteristics}

We summarise the analysis of our population of accretion-driven bursts in our Table 2 For each of the four models, it reports the number of the bursts with a specific magnitude (1-, 2-, 3-, and 4-mag) and also the total number of the bursts. It also indicates the maximum, minimum and mean values of the burst luminosity

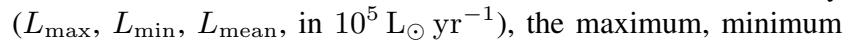
and mean values of the burst peak accretion rate through the sink cell $\left(\dot{M}_{\max }, \dot{M}_{\text {min }}, \dot{M}_{\text {mean }}\right.$, in $\left.\mathrm{M}_{\odot} \mathrm{yr}^{-1}\right)$ and the maximum, minimum and mean values of the burst duration $\left(t_{\mathrm{bst}}^{\max }, t_{\mathrm{bst}}^{\min }, t_{\mathrm{bst}}^{\operatorname{mean}}\right.$, in yr), respectively. Finally, it provides the integrated duration of the bursts $t_{\mathrm{bst}}$ (in yr), i.e., the time that the protostar in each model spends in the burst phase during the initial $60 \mathrm{kyr}$ of evolution. For each cut-off magnitude, the bold line reports the averaged values of those quantities.

The number of low-intensity accretion-driven bursts is much higher than that of high-intensity bursts, e.g. the integrated number of 1-mag bursts in our set of models is $N_{\mathrm{bst}}=128$ while it is $N_{\text {bst }}=44$ and $N_{\text {bst }}=12$ for the 2- to 4-mag bursts, respectively. This is because the low-rate accretion variability, provoked by accretion of dense portions of spiral arms, is more frequent than the high-rate variability caused by the infalling gaseous clumps. Our simulations with $\beta=4 \%$ and $10 \%$ have more 1-, 2- and 3mag bursts than in our simulations with $\beta=5 \%$, however, the opposite trend is seen for the 4-mag bursts. Thus, our results do not support the interpretation of the simplistic picture of discs exhibiting an efficiency of the gravitational instability directly proportional to the initial spin of the pre-stellar core and gradually increasing with $\beta$. The peak luminosities of 1-mag bursts range from $L_{\text {min }}=0.054 \times 10^{5} \mathrm{~L}_{\odot}$ to $L_{\max }=15.1 \times 10^{5} \mathrm{~L}_{\odot}$, whereas they vary by 2 and 1 orders of magnitudes for our 2- and 3-mag bursts, exhibiting $L_{\min }=0.15 \times 10^{5}$ and $4.99 \times 10^{5} \mathrm{~L}_{\odot}$ to reach $L_{\max }=45.6 \times 10^{5}$ and $65.7 \times 10^{5} \mathrm{~L}_{\odot}$, respectively. Therefore, some 1-mag bursts can be more luminous (in bolometric luminosity) than some 2- or 3-mag bursts, depending on the corresponding pre-burst background stellar luminosity. The model Run-1-hr does not have 4-mag bursts because, due to timestep restrictions, we could not run it for longer integration times.

Figs. 4 to 6 show several correlation plots of the various burst characteristics of a homogeneous subset of three of our models $\left(r_{\text {in }}=20 \mathrm{au}\right)$. Fig. 4 displays the burst peak luminosity $L_{\text {burst }}$ (in $\left.L_{\odot}\right)$ as a function of the burst duration $t_{\mathrm{bst}}$ with a colour coding giving information about the accreted mass per burst (see panel (a), in $M_{\odot}$ ) and the peak accretion rate $\dot{M}$ (in $\mathrm{M}_{\odot} \mathrm{yr}^{-1}$ ) as a function of the burst duration $t_{\mathrm{bst}}$ with a colour coding giving information about the accreted mass per burst (see panel (b), in $M_{\odot}$ ). On each plots the symbols and their sizes are functions of the burst magnitude (smallest symbols for 1-mag bursts and largest symbols for 4-mag bursts) and specify the models, to which each burst belongs (circles for our Run-long-4\%, squares for Run-long-5\% and triangles for Run-long-10\%). The overplotted line is a fit of all bursts together.

Fig. 4 a,b show that 4-mag bursts have $L_{\text {tot }}>3 \times 10^{6} \mathrm{~L}_{\odot}$ and a duration time $<10 \mathrm{yr}$ while 1 -mag bursts are located in the region with $L_{\text {tot }}<3 \times 10^{6} \mathrm{~L}_{\odot}$ and $>4 \mathrm{yr}$. The same distribution appears regardless of the model conditions. The overplotted line fitting the burst population highlights this general trend - luminous bursts are shorter in duration, even if our bursts diversity

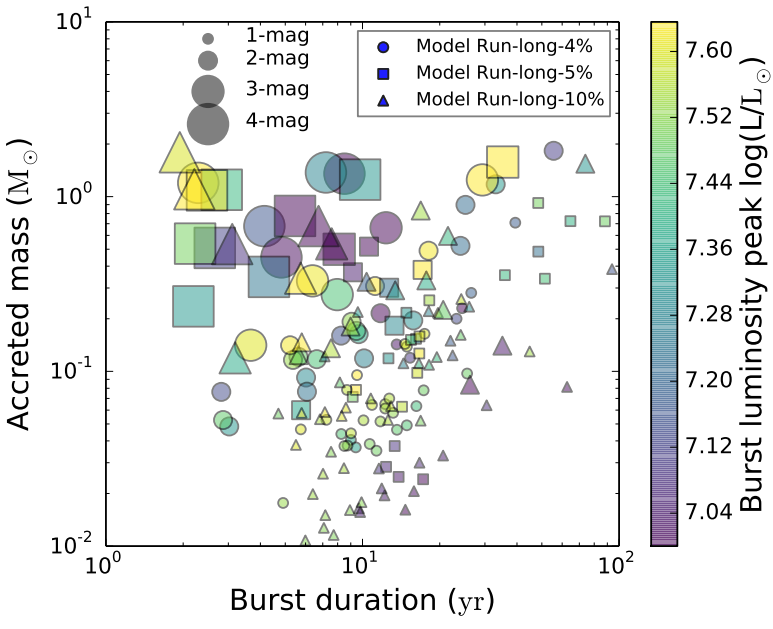

Figure 6. Correlation between burst duration versus accreted mass for each individual bursts, with colours representing the burst peak luminosity.

scatters in bolometric luminosity by spanning over several orders of magnitude at a given burst duration. Therefore, the burst peak luminosity (Fig. 4 a ) and maximal accretion rate (Fig. 4p) globally decrease with the burst duration (thick red lines in Fig 4). The more efficient mass-accreting bursts are mostly 3- and 4-mag, short-lived and some high-luminosity events or long-lived 1-mag bursts (see colour scale of Fig. 4 a). Such bursts are typically associated to the largest peak accretion rates, located in the short-duration and highluminosity region of the figure (Fig. 4p). This statistical discrepancy between the global correlation between peak accretion mass and peak accretion rate is already visible in our Table 2 and illustrates the variety of bursts duration produced for a given increase of the MYSOs's bolometric luminosity with respect to their pre-bursts luminosity.

Fig. 5 5 shows the accreted mass (in $\mathrm{M}_{\odot}$ as a function of the bursts peak accretion rate (in $\mathrm{M}_{\odot} \mathrm{yr}^{-1}$ ) and Fig. 5 plots the accreted mass as a function of the the bursts peak luminosity (in $\mathrm{L}_{\odot}$ ), respectively. Additionally, the colour coding indicates the burst peak luminosity (a) and the peak accretion rate during the bursts (b). Our models reveal a good correlation between the peak accretion rate and the accreted mass of their bursts (see thick red line). Fig. $5 \mathrm{p}$ is a consequence of the typical Gaussian-like shape of the accretion profiles, whose integral as a function of time (i.e. the accreted mass) is proportional to the peak accretion rate, whereas Fig. 5p is a natural outcomes of the used prescription to estimate $L_{\text {acc }} \propto \dot{M}$, low-luminosity deviations appearing because of occasional, rapid fluctuations of the stellar radius (Hosokawa \& Omukai 2009). Finally, Fig. 6plots for the burst duration (in yr) as a function of the accreted mass during the corresponding burst (in $\mathrm{M}_{\odot}$ ) with a colour coding giving information about the burst luminosity (in $\mathrm{L}_{\odot}$ ). This figure clearly illustrates our model predictions regarding luminosity bursts in MYSOs: more luminous bursts (3- and 4-mag) accrete more mass than their less luminous counterparts (1and 2-mag), although the former are shorter in duration than the latter. Accordingly, the different burst population do not overlap in the figure and organise in different, adjacent regions (Fig.6p). 
Table 3. Mass (in $\mathrm{M}_{\odot}$ ) and proportion (in \%) of final protostellar mass accreted as a function of the protostellar brightness at the moment of the accretion.

\begin{tabular}{|c|c|c|c|c|c|c|}
\hline Models & $M_{\star}$ & $\mathrm{L}_{\mathrm{tot}} \sim \mathrm{L}_{\mathrm{bg}}$ & $1-\operatorname{mag}$ & $2-$ mag & $3-$ mag & 4-mag \\
\hline \multicolumn{7}{|c|}{ Accreted mass $\left(\right.$ in $\left.\mathrm{M}_{\odot}\right)$} \\
\hline Run-long-4\% & 47.33 & 25.06 & 5.28 & 10.04 & 2.59 & 4.36 \\
\hline Run-long-5\% & 41.57 & 21.48 & 5.48 & 4.97 & 5.20 & 4.44 \\
\hline Run-long-10\% & 34.15 & 19.35 & 6.39 & 3.66 & 0.93 & 3.82 \\
\hline Run-hr & 20.07 & 15.25 & 1.13 & 1.88 & 1.74 & 0.07 \\
\hline $\operatorname{Mean}\left(r_{\mathrm{in}}=20 \mathrm{au}\right)$ & 41.02 & \multicolumn{5}{|c|}{ (vin } \\
\hline Run-without & 24.76 & 18.64 & 3.01 & 2.46 & 0.63 & 0.00 \\
\hline Run-with & 24.35 & 15.46 & 3.76 & 3.63 & 1.19 & 0.29 \\
\hline \multicolumn{7}{|c|}{ Proportion of final protostellar mass (in \%) } \\
\hline Run-long- $4 \%$ & 100 & 52.94 & 11.17 & 21.21 & 5.47 & 9.21 \\
\hline Run-long-5\% & 100 & 51.66 & 13.18 & 11.95 & 12.51 & 10.70 \\
\hline Run-long-10\% & 100 & 56.65 & 18.70 & 10.73 & 2.74 & 11.18 \\
\hline Run-hr & 100 & 75.97 & 5.60 & 9.36 & 8.70 & 0.37 \\
\hline $\operatorname{Mean}\left(r_{\text {in }}=20 \mathrm{au}\right)$ & \multicolumn{5}{|c|}{ (25) } & 10.36 \\
\hline Run-without & 100 & 75.30 & 12.19 & 9.93 & 2.55 & 0.00 \\
\hline Run-with & 100 & 63.49 & 15.45 & 14.94 & 4.89 & 1.21 \\
\hline
\end{tabular}

\section{THE EFFECT OF STELLAR MOTION}

The role of stellar motion on the disc dynamics and bursts properties is examined in this section. We first introduce the mechanism responsible for stellar motion and its implementation in our code, and then compare two simulations of equivalent initial conditions and included physics, but considered without and with stellar wobbling.

\subsection{Stellar inertia and its implementation in the PLUTO code}

When hydrodynamical simulations of circumstellar discs are run using a curvilinear coordinate system, the sink cell representing the star and its direct surrounding is attached to the origin of the computational domain. The natural response of the star to the changes in the mass distribution of its circumstellar disc, when the disc barycenter does not coincide with the origin of the domain, is therefore neglected. Taking stellar motion into account may become important when the initial axisymmetry of the rigidly-rotating prestellar core is broken by the development of dense spiral arms and heavy gaseous clumps in the disc and their gravitational pull on the star is not mutually canceled. This mechanism has been first revealed in the analytical study on eccentric gravitational instabilities in discs around low-mass protostars of Adams et al. (1989). It is intrinsically taken into account in Lagragian simulations such as the smooth hydrodynamical models (SPH) of, e.g. Bonnell \& Bate (1994); Rice et al. (2005); Meru \& Bate (2010); Lodato \& Clarke (2011) as well as in simulations performed with three-dimensional Cartesian codes, see e.g. Krumholz et al. (2007); Kratter et al. (2010); Commerçon et al. (2011); Klassen et al.(2016); Rosen et al. (2016). Regály \& Vorobyov (2017) and Vorobyov \& Elbakyan (2018) studied the effect of stellar wobbling on the disc evolution and migration of gaseous clumps and protoplanets using grid-based codes in the polar coordinates. They found that stellar motion may affect notably the migration speeds of gaseous clumps and protoplanets in the disc, but the final disc masses and sizes and also disc propensity to fragment were affected to a lesser extent. Since highmass protostars are surrounded by massive discs (Johnston et al. 2015. Ilee et al. 2016, Forgan et al. 2016, Cesaroni et al. 2017) in which heavy substructures (gaseous clumps, low-mass compan- ions and/or asymmetric spiral arms) can form by fragmentation in the disc (Meyer et al. 2018), the question is therefore how much those asymmetries may affect the total gravitational potential of the protostar-disc system during their rather short pre-main-sequence phase $(\sim$ kyr $)$, see Section 4.2 .

Our implementation of the stellar motion into the PLUTO code for the spherical coordinate system consists in calculating the resultant gravitational force applied by the disc to the star using Cartesian projections and it is inspired from Hirano et al. (2017). The gravitational force applied by the massive disc onto the growing protostar reads,

$$
\delta \vec{F}_{\mathrm{disc} / \star}=-G \frac{\delta M_{\mathrm{disc}}(r) M_{\star}}{r^{2}} \vec{e}_{\mathrm{r}},
$$

where $\delta M_{\text {disc }}(r)$ is the mass of a grid zone located in the disc, $r$ is the distance of the considered cell to the central protostar and $\vec{e}_{\mathrm{r}}$ is the unit vector in the radial direction. The projection of the force

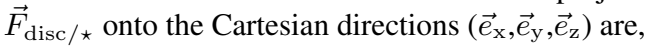

$$
\begin{aligned}
& \delta F_{\mathrm{disc} / \star}^{x}=\delta \vec{F}_{\mathrm{disc} / \star}^{x} \cdot \vec{e}_{\mathrm{x}}=\delta\left|\vec{F}_{\mathrm{disc} / \star}\right| \cos (\phi) \sin (\theta), \\
& \delta F_{\mathrm{disc} / \star}^{y}=\delta \vec{F}_{\mathrm{disc} / \star}^{y} \cdot \vec{e}_{\mathrm{y}}=\delta\left|\vec{F}_{\mathrm{disc} / \star}\right| \sin (\phi) \sin (\theta),
\end{aligned}
$$

and,

$$
\delta F_{\mathrm{disc} / \star}^{z}=\delta \vec{F}_{\mathrm{disc} / \star}^{z} \cdot \vec{e}_{\mathrm{z}}=\delta\left|\vec{F}_{\mathrm{disc} / \star}\right| \cos (\theta),
$$

where $\phi$ and $\theta$ represent the azimuthal and polar angles, respectively. We integrate the Cartesian components of the gravitational backaction of the circumstellar disc onto the protostar. Therefore, the Cartesian components of the total force $\vec{F}_{\mathrm{disc} / \star}$ read,

$$
\begin{aligned}
& F_{\mathrm{disc} / \star}^{x}=\int_{\mathrm{disc}} \delta F_{\mathrm{disc} / \star}^{x}, \\
& F_{\mathrm{disc} / \star}^{y}=\int_{\mathrm{disc}} \delta F_{\mathrm{disc} / \star}^{y},
\end{aligned}
$$

and

$$
F_{\mathrm{disc} / \star}^{z}=\int_{\mathrm{disc}} \delta F_{\mathrm{disc} / \star}^{z},
$$


where the disc is evaluated as the gas that lies within the innermost envelope located at radii $\leqslant 3000$ au of the computational domain (Meyer et al. 2018). Finally, we recover,

$$
\vec{F}_{\text {disc } / \star}=\sqrt{\left(F_{\text {disc } / \star}^{x}\right)^{2}+\left(F_{\text {disc } / \star}^{y}\right)^{2}+\left(F_{\text {disc } / \star}^{z}\right)^{2}} \vec{e}_{\mathrm{r}},
$$

which is the total force exerted by the disc onto the star. In our midplane-symmetric picture,

$$
F_{\text {disc } / \star}^{z}=0
$$

and $\vec{F}_{\text {disc/^ }}$ reduces to,

$$
\vec{F}_{\mathrm{disc} / \star}=\sqrt{\left(F_{\mathrm{disc} / \star}^{x}\right)^{2}+\left(F_{\mathrm{disc} / \star}^{y}\right)^{2}} \vec{e}_{\mathrm{r}} .
$$

As we keep the protostar fixed to the origin of the computational domain, the effective force that we apply to each grid cell is,

$$
\vec{F}_{\text {wobbling }}=\vec{F}_{\star / \text { disc }}=-\vec{F}_{\text {disc } / \star},
$$

that has the effect to translate the disc solution around the star by the opposite vector of the force exerced by the disc onto it. The effect of the wobbling force is finally introduced as an additional acceleration term,

$$
\vec{g}^{\prime}=\frac{\vec{F}_{\text {wobbling }}}{M_{\star}},
$$

in the gravity solver of the PLUTO code. In spherical coordinates, the different components of $\vec{g}^{\prime}$ read,

$$
\begin{array}{r}
g_{r}^{\prime}=\frac{1}{M_{\star}}\left[F_{\mathrm{disc} / \star}^{x} \sin (\theta) \cos (\phi)+F_{\mathrm{disc} / \star}^{y} \sin (\theta) \sin (\phi)\right. \\
\left.+F_{\mathrm{disc} / \star}^{z} \cos (\theta)\right], \\
\vec{g}_{\phi}^{\prime}=\frac{1}{M_{\star}}\left[F_{\mathrm{disc} / \star}^{x} \cos (\theta) \cos (\phi)+F_{\mathrm{disc} / \star}^{y} \cos (\theta) \sin (\phi)\right. \\
\left.-F_{\mathrm{disc} / \star}^{z} \sin (\theta)\right],
\end{array}
$$

and,

$$
\vec{g}_{\theta}^{\prime}=\frac{1}{M_{\star}}\left[-F_{\mathrm{disc} / \star}^{x} \sin (\phi)+F_{\mathrm{disc} / \star}^{y} \cos (\phi)\right],
$$

respectively. As described by Regály \& Vorobyov (2017), this method induces boundary effects at the outer extremities of the computational domain, when the stellar motion is so pronounced that the corresponding translation of the whole solution by $\vec{F}_{\text {wobbling }}$ gradually provokes cavities that open at the outer edges of the pre-stellar core. This is an issue meriting future numerical investigations and code development, however, it does not affect our comparison tests because we run simulations with high-mass cores that collapse faster $(\sim 40 \mathrm{kyr})$ than in the context of solar-mass pre-stellar cores. In the section below, we compare two simulations of gravitational collapse and disc formation of similar initial conditions but considered with and without stellar motion.

\subsection{Effects of stellar wobbling on mass accretion}

To compare the effects of stellar motion on the accretion disc and protostellar properties, we carry out two additional simulations initialised with a $100 \mathrm{M}_{\odot}$ pre-stellar core, rigidly-rotating with $\beta=4 \%$ and having a sink cell with a radius of $r_{\text {in }}=12 \mathrm{au}$. We use a small sink cell radius in order to follow the clumps migration at radii $\leqslant 20 \mathrm{au}$. This, however, reduces the timestep of the simulations and the timescale over which we can perform the model runs (see Run-1-hr). Hydrodynamics and radiation transport are treated as in the other runs of this study (see Table 1 . We additionally include the effects of the stellar motion in one run (Run-with), whereas the other simulation neglects it (Run-without). Fig. 7 a shows the evolution of the accretion rate onto the protostar (in $\mathrm{M}_{\odot} \mathrm{yr}^{-1}$ ) in our comparison simulations as a function of time (in yr). The Figure distinguishes between the model without (red solid line) and with (blue dashed line) stellar wobbling. The mass of the protostar is also plotted for the run without (thick dotted black line) and with (thick solid black line) wobbling. As the initial conditions are similar, the time of the free-fall collapse is the same in both runs $(\approx 12 \mathrm{kyr})$ and moderate accretion variability onto the MYSOs develops in the accretion flow similarly after the onset of the disc formation phase. In both cases (without and with stellar motion), the accretion rate is interspersed with episodic accretion spikes arising in addition to the baseline accretion rate of a few $\times 10^{-4} \mathrm{M}_{\odot} \mathrm{yr}^{-1}$. This demonstrates that, the stellar motion does not prevent the production of accretion-driven outbursts through the disc-fragmentation-based mechanism described in Meyer et al. (2017) and Meyer et al. (2018) and also has little effect on the final mass of the MYSOs $\left(\approx 25 \mathrm{M}_{\odot}\right)$.

The thin red lines in Fig. 7 p,c show the total luminosity of the MYSOs, i.e. the sum of the photospheric and accretion luminosity (in $L_{\odot}$ ) as a function of time (in kyr) of both comparison models. In addition, the black solid lines show the background luminosity $L_{\mathrm{bg}}$, while the dotted thin blue line, dashed dotted orange line and dashed violet lines show the limits of the 1-, 3- and 4-magnitudes cutoffs with respect to $L_{\mathrm{bg}}$, respectively. The overplotted dots represent the peak accretion rates for each individual burst, the peak luminosity of which is at least 2.5 times brighter than their quiescent pre-flare luminosity and they are coloured as a function of the peak accretion rate, shown in the $0.001-0.1 \mathrm{M}_{\odot} \mathrm{yr}^{-1}$ range. As in our other models, the time evolution of the luminosity is a direct function of the mass accretion rate (Fig. 7 p). In both comparison models (without and with wobbling), variations in the luminosity begins $t \approx 12 \mathrm{kyr}$ when the accretion luminosity ceases to be negligible as compared to the photospheric luminosity. The protostellar accretion rate history then further affects the total luminosity of the protostars by producing variations of increasing intensity in their lightcurves, according to the mechanism described above in this work and in Meyer et al. (2017). Moreover, we recover the initial development of mild accretion-driven bursts with a peak accretion rate $\leqslant 10^{-3} M_{\odot} \mathrm{yr}^{-1}$ at $\approx 17 \mathrm{kyr}(1,2$-mag bursts) that precede stronger flares with a peak accretion rate $\geqslant 10^{-1} M_{\odot} \mathrm{yr}^{-1}$ at times $\geqslant 29$ yr (3,4-mag bursts). We report in Table 2 the bursts statistics and properties (peak luminosity, peak accretion rate, duration).

The similitude between the two comparison models goes beyond a simple general, qualitative aspect of their accretion rate histories and luminosity curves, but it also concerns the properties of the bursts themselves. The number of bursts is similar in both models. For example, we obtain the same number of bursts for the 2- and 3-mag flares and find only a slight difference for the 1-mag bursts (9 in comparison to 6 flares when wobbling is included. We also note the presence of a 4-mag burst in the model with stellar wobbling. In both cases, the bursts have durations of the same order of magnitude, with $t_{\mathrm{bst}}^{\text {mean }}=38 \mathrm{yr}$ (without) and $29 \mathrm{yr}$ (with), $t_{\mathrm{bst}}^{\text {mean }}=18 \mathrm{yr}$ (without) and $21 \mathrm{yr}$ (with) and $t_{\mathrm{bst}}^{\text {mean }}=5 \mathrm{yr}$ (without) and $9 \mathrm{yr}$ (with), for the 1-, 2- and 3-mag bursts, respectively. Additionally, one can note that the total time the model with stellar wobbling spends in the burst phase $(300 \mathrm{yr}$, representing $\approx$ $0.75 \%$ of the modelled $40 \mathrm{kyr}$ ) is slightly shorter than that without wobbling ( $438 \mathrm{yr}$, representing $\approx 1.1 \%$ of the modelled $40 \mathrm{kyr}$ ) 

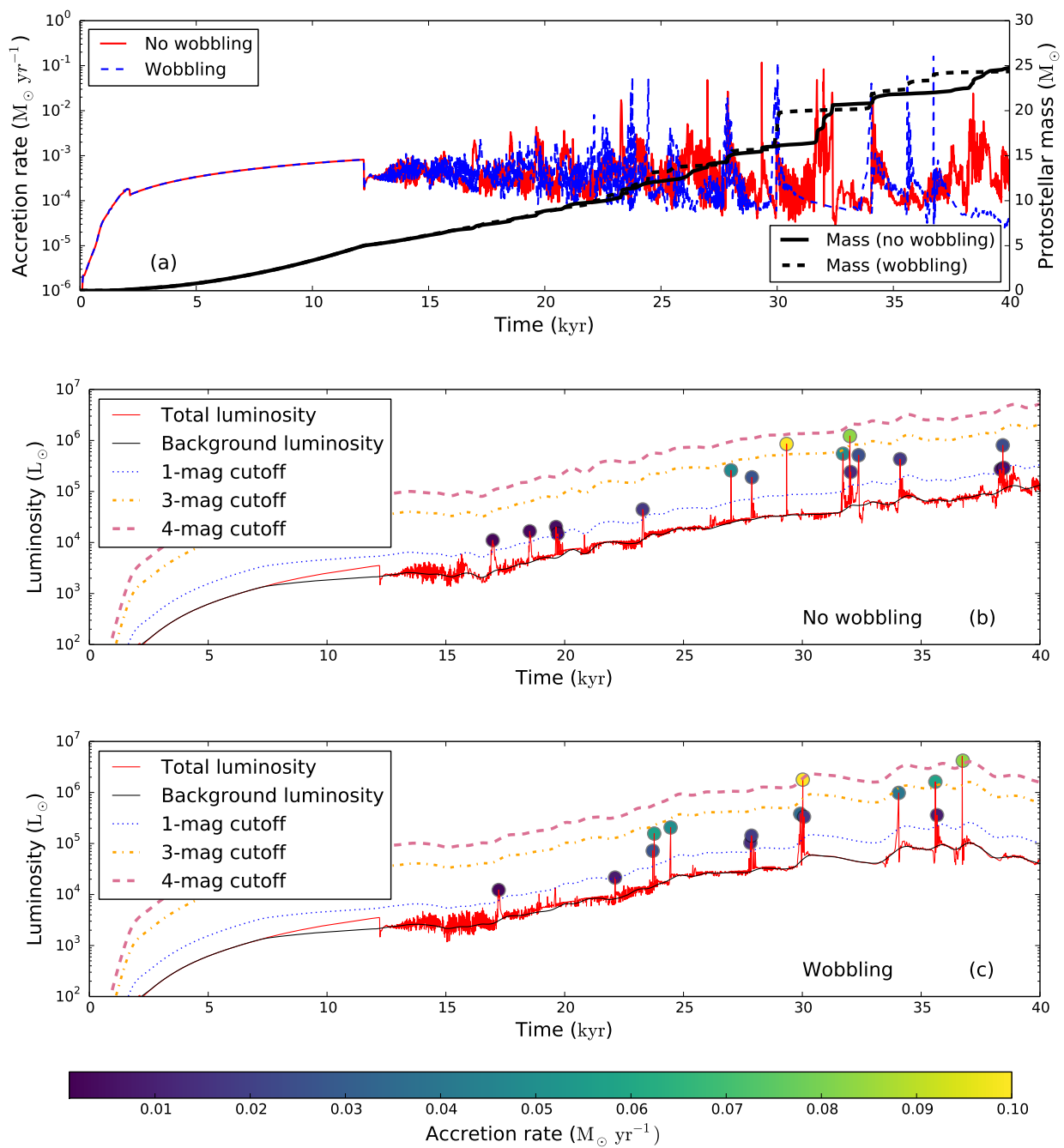

Figure 7. Comparison of mass accretion rates and luminosities in our comparison model ( $\beta=4 \%$ and $r_{\text {in }}=12$ au) with and without stellar wobbling. Panel (a) shows the mass accretion rate (thin lines, in $\mathrm{M}_{\odot} \mathrm{yr}^{-1}$ ) and protostellar masses (thick lines, in $\mathrm{M}_{\odot}$ ) in our simulations without (solid lines) and with (dashed lines) stellar wobbling. Panels (b) and (c) present the total luminosities (thin red solid lines, in kyr), background luminosities (thin solid black lines) and luminosity cutoffs (the dotted and dashed lines) for the model without stellar wobbling (middle panel) and with stellar wobbling (bottom panel). The color bar indicates the accretion rate for the filled circles in panels (b) and (c).

while the accrete mass is larger (see below). The mean luminosity and mean peak accretion rates of the two models are equivalent. For example, for the 2 -mag bursts $L_{\text {mean }} \approx 4.64 \times 10^{5}$ and $\dot{M}_{\text {mean }} \approx 0.0343 \mathrm{M}_{\odot} \mathrm{yr}^{-1}$ in the model without stellar wobbling and $L_{\text {mean }} \approx 4.12 \times 10^{5} \mathrm{~L} \odot \mathrm{yr}^{-1}$ and $\dot{M}_{\text {mean }} \approx 0.0397 \mathrm{M}_{\odot} \mathrm{yr}^{-1}$ in the model with stellar wobbling. Moreover, both models exhibit bursts happening either isolated or in clusters, which strengthens the similitude between the two comparison simulations.

More importantly, the analysis of the fractions of final mass accreted as a function of the protostellar luminosity (Table 3. shows that the disc response to stellar motion does not dramatically affect our conclusions in terms of the accreted mass throughout the eruptive episodes. In both comparison models, the star accretes $\approx 24 \mathrm{M}_{\odot}$ over $40 \mathrm{kyr}$ and gains $18.64 \mathrm{M}_{\odot}(\approx 75 \%)$ and $15.46 \mathrm{M}_{\odot}(\approx 63 \%)$ of its final mass when $L_{\mathrm{tot}} \simeq L_{\mathrm{bg}}$, which is in accordance with what was found in our model Run-1-hr that was modelled over a similar timescale ( $35 \mathrm{kyr})$. Our results show that the model with stellar wobbling accretes more mass through bursts as compared to the simulation without wobbling, especially during the 2- and 3-mag bursts for which the accreted mass is doubled compared to the model without wobbling. Interestingly, our model with wobbling exhibits a 4-mag burst while the model without stellar wobbling does not (at least during the first $40 \mathrm{kyr}$ ), as our simulation Run-long- 4 with $\beta=4 \%$ but $r_{\text {in }}=20$ au has its first 4 -mag burst at $\approx 43 \mathrm{kyr}$ ). This mean that our models with stellar motion form massive clumps earlier in the evolution that the models without wobbling. We conclude that the burst characteristics are only weakly sensitive to stellar motion.

\section{DISCUSSION}

In this section, we present the limitation and caveats of our model, estimate the burst occurence during the early protostellar formation 


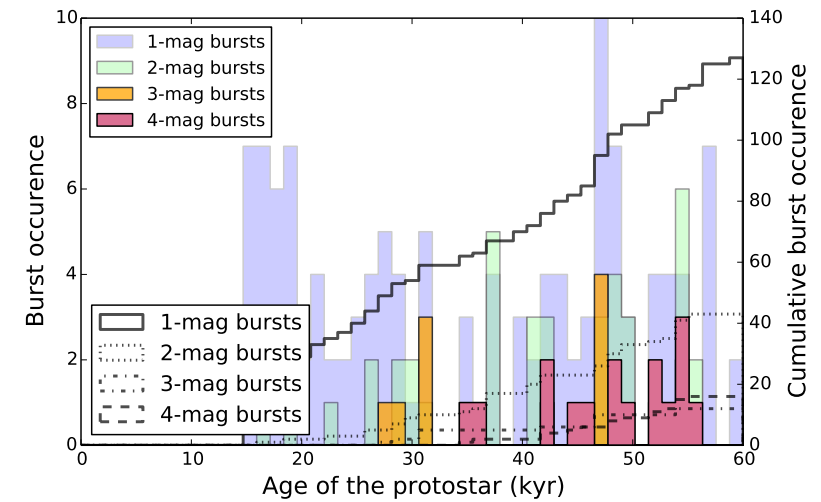

Figure 8. Bursts occurence as a function of the age of the protostar.

phase and discuss the connection between bursts and protostellar mass growth. Furthermore, we review our knowledge of observations of several monitored bursts of massive protostars.

\subsection{Model limitation}

The assumptions of our models are globally similar to those of Meyer et al. (2017), where we describe several improvements which could be added to our model. These improvements include making the initial conditions more realistic and by improving the grid geometry/resolution. Indeed, our parent pre-stellar cores are assumed to be initially spherically-symmetric, while high-mass stars also form in filamentary structures (see, e.g. Banerjee et al. 2006). The midplane-symmetry imposed in our simulations could be improved in favour of full three-dimensional protostellar disc models. Moreover, although the logarithmically-expanding grid in the radial direction permits fulfilling the Truelove criterion (Truelove et al. 1998), which warrants the correct treatment of selfgravity within the disc, a full convergence of the entire disc structure is not reached (if possible at all) and higher resolution models would allow us to investigate the clumps trajectories in the very inner disc. Nevertheless, we show in Meyer et al. (2018) that our accretion rate histories qualitatively converge as the numerical resolution is increased.

As an improvement compared to the previous works in this series, the longer integration times of our simulations up to $60 \mathrm{kyr}$ follow better the disc evolution, the aftermath of gravitational instabilities and/or clump migration in the protostellar lightcurves. Even longer runs should be conceived, e.g. in order to determinate the zero-age-main sequence of the MYSOs and see whether the discs modify their morphology and/or orientation as around evolved lowmass protostars (Matsumoto et al. 2017). Additionally, the huge parameter space should be explored within a large grid of simulations. One should nevertheless keep in mind that such improvements will make the simulations more computationally-intensive, which is at the moment not affordable and remains out of reach of modern supercomputing capabilities. Apart from the above mentioned caveats, the sink cell size may affect our results. A smaller size of the sink cell allows to further follow the clump migration, internal evolution and probable stretching and fragmentation. Using a smaller sink cell dramatically decreases the time-step of the simulations, which in turn makes them even more costly. A more thorough discussion of our model limitations can be found in Meyer et al. (2018).

\subsection{Burst occurence}

Fig. 8 plots the burst occurrence as a function of the age of the protostar. More specifically, the histograms report the number of the bursts per time interval for different burst magnitudes (1-, 2, 3-, and 4-mag). The lines show the cumulative burst occurrence for different burst magnitudes as a function of protostellar age. The colour coding is similar as in Figs. 4/6 As indicated in Table 2. our MYSOs burst incidence is 128, 44, 12 and 17 for 1-mag (solid line), 2-mag (dotted line), 3-mag (dashed dotted line) and 4-mag bursts (dashed line), respectively. Initially, the bursts are mostly 1mag bursts up to $\approx 30 \mathrm{yr}$, although run-long-5 has already some 3 -mag bursts at a time $\approx 27 \mathrm{kyr}$ (Fig. 2p) and run-long-10 shows 2 -mag bursts at a time $\approx 20 \mathrm{kyr}$ (Fig. 2F). As the protostars evolve towards the main sequence phase, the burst activity strongly increases, with a accumulation of 3-mag and 4-mag bursts starting from $\approx 40 \mathrm{kyr}$, when our protostars experience a period of rapid and violent eruption (Fig. 2a) during which the probability to observe such phenomenon is the larger.

Fig. 9 presents the number of bursts and the cumulative number of bursts as a function of the burst duration (a), accreted mass per bursts (b), burst peak luminosity (c) and burst accretion rate (d). The burst duration remains within the $2-100 \mathrm{yr}$ limits, with a moderate dependence on the initial pre-stellar core spin, as a result of the convergence of our simulations. A higher-resolution model run-hr has about twice more bursts as run-long-4, despite similar initial conditions since the former better resolves and further follows the second collapse of the migrating clumps because of its smaller sink cell radius $r_{\text {in }}$. As the higher-magnitude bursts are caused by more massive and compact clumps, these bursts are evidently shorter in time $\left(t_{\mathrm{bst}}^{\text {mean }} \approx 4 \mathrm{yr}\right.$ ) than lower-magnitude ones $\left(t_{\mathrm{bst}}^{\text {mean }} \approx 27 \mathrm{yr}\right.$, which are caused by more diffuse and extended clumps. The stronger the bursts the smaller their duration and therefore the less probable it is to observe such events of MYSOs, which raises the question of their possible observability. Our lower amplitude burst (1- and 2-mag) characteristics are nevertheless in rather good agreement with observations of bursts from massive protostars (Section 5.4).

The accreted mass spans a range from a few Jupiter masses to a few solar masses and the accreted mass during the outburst of S255IR NIRS 3 lies in this range of values (Caratti o Garatti et al. 2017). This distribution of accreted masses reflects the apparent variety of disk fragments, e.g. collapsing clumps or overdense portion of spiral arms formed via disk gravitational instability and fragmentation and migrated in the inner disc region. The strongest accretion events may be associated to the formation of low-mass companions to the central MYSOs. In Meyer et al. (2018), within the so-called disc fragmentation scenario for the formation of close/spectroscopic binaries around proto-OB stars, we showed the formation possibility of such objects, when migrating massive clumps may both lose their diffuse envelope and further contract into their center to form a secondary low-mass protostellar core. Such an event would then produce a companion and a strong (4-mag) burst from a single infalling dense clump. Since we do not consider this effect in the current study, the actual bursts may be of slightly lower amplitude than our model predicts. Interestingly, the number of 4-mag bursts predicted in our model (from 5 to 8 ) is consistent with the occurrence of close binaries around OB stars (Chini et al.2012; Mahy et al. 2013, Kobulnicky et al. 2014).

The burst luminosity distribution clearly shows that 1-mag and 2-mag bursts are by far the more frequent (Fig.9 9 ), with a luminosity peak $\approx 10^{5}-10^{6} \mathrm{~L} \odot$. Other bursts of higher luminosity are not 

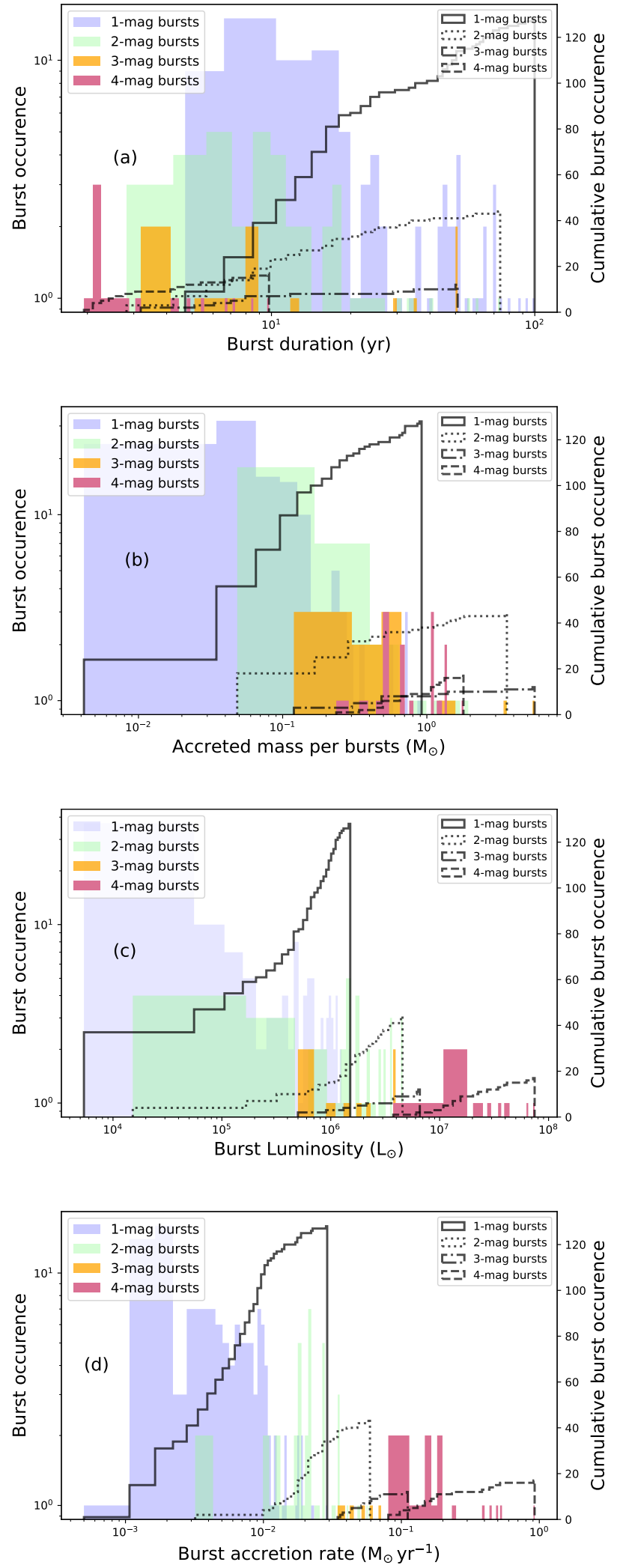

Figure 9. Burst occurence as a function of the burst duration (a), accreted mass per burst (b), burst peak luminosity (c) and burst peak accretion rate (d). as common as their fainter counterparts, and, if some 3-mag bursts can reach luminosity peaks $\geqslant 10^{6} \mathrm{~L}_{\odot}$, only 4-mag bursts can reach luminosities $\geqslant 10^{7} \mathrm{~L}_{\odot}$. As was stated above, these strong bursts might be simultaneously associated with the formation of binaries and, thus, may be of slightly fainter intensity. Consequently and given their rareness, one should not expect to observe bursts from MYSOs more luminous than $\sim 10^{7} \mathrm{~L} \odot$, if at all. The last histogram (Fig. 9d) plots the burst peak accretion rate distribution. It underlines the correlation between $L_{\max }$ and $\dot{M}_{\max }$, already depicted in Fig. 5p: the stronger the burst the higher the peak accretion rate of circumstellar material. Most bursts peak at $\simeq 10^{2} \mathrm{M}_{\odot} \mathrm{yr}^{-1}$, while only a minor fraction of them exceed $\simeq 10^{-1} \mathrm{M}_{\odot} \mathrm{yr}^{-1}$.

\subsection{Protostellar mass growth by episodic accretion}

Fig. 10 plots the mass evolution of the protostar (in $\mathrm{M}_{\odot}$ ) in our Run-long-4 model as a function of time (in yr). The Figure distinguishes between the total accreted mass of the protostar (thick solid red line) and the mass accreted when the protostar is in the quiescent phase (thin solid black line), expericing 1-mag (thin dotted blue line), 2-mag (thin dashed green line), 3-mag (solid orange line) and 4-mag (thin dotted dashed purple line) bursts. During the freefall gravitational collapse and up to the end of the smooth accretion phase, the protostar grows exclusively by quiescent accretion driven by gravitational torques that are generated by anisotropies in the inner disc flow. In this case, variations in stellar brightness remains smaller than 1-mag, i.e. increase by a maximal factor of $\approx 2.5$ compared to $L_{\mathrm{bg}}$. When the young star enters the high-mass regime $\left(\geqslant 8 \mathrm{M}_{\odot}\right.$, orange circle), no clear bursts has yet happened, although accretion bursts start to occur in the models with a higher $\beta$ (Fig. 3). During the burst phase of accretion, more and more mass is accreted while the protostar experiences $\geqslant 2$-mag bursts. As was already discussed in our Table 2 the higher the burst intensity, the later the burst appear and the rarer they are, as testifies the decreasing number of step-like escalation of the mass histories at times $\geqslant 35 \mathrm{kyr}$. The Figure also depicts the larger proportion of the 1and 2-mag bursts compare to the less frequent 3-and 4-mag ones. All our models have similar behaviour.

In Table 3 we summarise the stellar mass accreted in the quiescent mass and during the bursts (for bursts of different magnitude) and express the results in terms of mass and percentage of the final protostellar mass, respectively. Our model Run-long4 accretes $\approx 25.06 \mathrm{M}_{\odot}$ in the quiescent phase and $\approx 5.28 \mathrm{M}_{\odot}$, $\approx 10.04 \mathrm{M}_{\odot}, \approx 2.59 \mathrm{M}_{\odot}$ and $\approx 4.36 \mathrm{M}_{\odot}$ while experiencing 1- to 4-mag bursts, respectively. By performing an average of the different values over our models with $r_{\text {in }}=20 \mathrm{AU}$, we find that protostars gain, during their early $60 \mathrm{kyr}$, about $5.72,6.22,2.91$ and $4.21 \mathrm{M}_{\odot}$ while experiencing 1-mag, 2-mag, 3-mag, and 4-mag bursts, respectively. In other words, a fraction of $\approx 53.75 \%$ of the final mass is gained by quiescent accretion (when $L_{\text {tot }} \simeq L_{\mathrm{bg}}$ ) while about $14.35 \%, 14.63 \%, 6.91 \%$ and $10.36 \%$ of the final mass is accreted during 1-mag to 4-mag accretion-driven events. Over the first $60 \mathrm{kyr}$ of their life, as a direct consequence of the rareness of the strongest flares, the total time the MYSOs spend in 1 -mag bursts $\left(\approx 1.27 \%\right.$ of $\left.t_{\text {end }}\right)$ is longer than the cumulative time of all higher intensity bursts $\left(\approx 0.43 \%\right.$ of $\left.t_{\text {end }}\right)$, which implies a very rare observation probability. The total burst time $\approx 1027 \mathrm{yr}$ is $\approx 1.7 \%$ of the $60 \mathrm{kyr}$, but it permits the accretion of a significative fraction of about half $(\approx 46.25 \%)$ of their final mass, which demonstrates the importance and non-negligibility of the accretion bursts in the understanding of the early evolution and mass growth of MYSOs. 


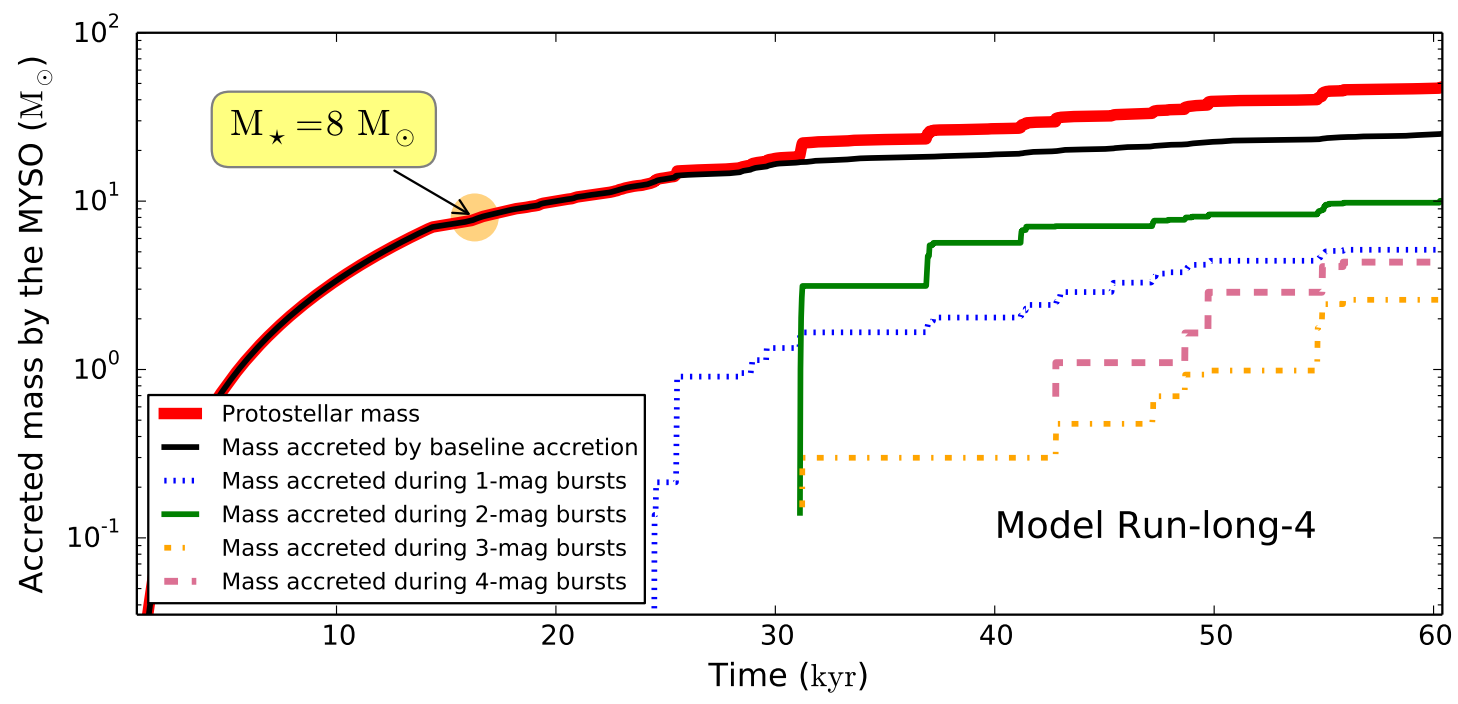

Figure 10. Protostellar mass growth as a function of the star's total luminosity.

\subsection{Comparison with observations}

The discovery and analysis of episodic bursts from massive protostars is hampered by various circumstances. According to the initial stellar mass function, these objects are much rarer than their lowmass counterparts and thus, on average, more distant. Their protostellar evolution occurs at much higher pace compared to low-mass young stellar objects, so they remain deeply embedded during most of this evolutionary period. This renders the discovery of burstinduced photometric variability impossible at optical and difficult at infrared wavelengths in contrast to smaller-mass YSOs (Scholz et al. 2013). Since the most massive young star dominates the emission of embedded clusters, attempts were made to trace its variability in scattered light (Stecklum et al.|2017). A remedy comes from the frequent association of high-mass young stellar objects with masers. In particular methanol Class II masers are a reliable signpost of massive star formation (Breen et al. 2013). These masers arise from molecules which are released from the ice mantles of grains to the gas phase by heating of the dust which surrounds the massive protostar. The maser excitation can be collisional, e.g. in shocks of bipolar outflows, or due to radiative pumping, as in the case of the $6.7 \mathrm{GHz}$ Class II methanol masers (Sobolev et al. 1997). Thus, the temporary increase in luminosity due to an accretion burst may cause flares of radiatively pumped masers. However, other circumstances affect the maser strength as well. In fact, many of these masers show pronounced variability which can be even periodic (Goedhart et al. 2003). The non-linear maser response in the non-saturated regime makes it difficult to judge the increase of the mid-IR radiation. Nevertheless, to some extent, the variability of Class II methanol masers may be considered to reflect luminosity variations of MYSOs (Szymczak et al. 2018). Indeed, recent observations confirmed this view for two objects, described in the following.

First evidence for bursts from massive protostars was gained from the case of V723 Carinae (Tapia et al. 2015b a), a $\sim 10 \mathrm{M}_{\odot}$ MYSO with a post-burst luminosity of $4 \times 10^{3} \mathrm{~L}_{\odot}$. It was fainter than $K_{s} \simeq 16.6$ in 1993 and found to be three magnitudes brighter in 2003 (Tapia et al. 2006). The object reached its maximum $K_{s}$ brightness of 12.9 in 2004 February and shows erratic variability of up to $\Delta K_{s}=2$ since then. Its infrared colours resemble those of Class I objects and indicate the presence of very substantial extinction. Because of the sparse pre-burst IR photometry, no conclusion on the luminosity increase during the burst could be drawn. At the time of its discovery, V723 Carinae was the most luminous, most massive, most deeply embedded, and possibly youngest eruptive young variable.

This situation changed in 2015 when a flare of the $6.7 \mathrm{GHz}$ methanol maser in the S255-IR star forming region was announced (Fujisawa et al. 2015). Immediate NIR follow-up imaging disclosed that NIRS3, a $\sim 20 \mathrm{M}_{\odot}$ MYSO representing the most luminous member of the embedded cluster, brightened by $\Delta K_{s}=2.5$ compared to the latest archival data from 2009 (Stecklum et al. 2016). A thorough investigation of the NIRS3 burst was possible thanks to the observational advancement since the V723 Carinae event and the availability of spectroscopic as well as photometric pre-burst data. Major results include the discovery of spectral features which are typical for young low-mass eruptive variables - however three orders of magnitude stronger, the discovery of the light echo from the burst which allows to reconstruct its history, and an increase of the bolometric luminosity from $\left.2.9 \pm_{0.7}^{1}\right) \times 10^{4} \mathrm{~L}_{\odot}$ to $\left(1.6 \pm_{0.3}^{0.4}\right) \times 10^{5} \mathrm{~L} \odot$ which points to an accretion rate of $(5 \pm 2) \times 10^{-3} \mathrm{M}_{\odot} \mathrm{yr}^{-1}$ (Caratti o Garatti et al. 2017). The burst was accompanied by a spatial rearrangement of 6.7 GHz methanol masers (Moscadelli et al. 2017) at subluminal speed (Stecklum et al. 2018) as well as enhanced outflow activity traced by an increase of the radio continuum emission, delayed by a about one year (Cesaroni et al. 2018).

Notably, there is evidence from the distribution of molecules around NIRS3 that its nearest vicinity may be considerably inhomogeneous (Zinchenko et al. 2015, 2017) and may be subject to gravitational instability. Various authors reported about four bursts from NIRS3, including the recent one, within the last $\sim 7000$ years (Wang et al. 2011, Zinchenko et al. 2015, Burns et al. 2016). The arrangement of bow shocks in the outflows in this region seems to support multiple outbursts (Zinchenko et al. 2015), although most recent ALMA data shows that the situation is complicated and the youngest of the bow shocks is produced by another source in the region, SMA2 (Zinchenko et al.2018). 
This seems to indicate that 2015 burst is part of a series of eruptive events and, probably, more are to come in the future. Such remark can be applied to the other observed bursts from MYSOs, and, more generally, by analogy with low-mass star formation, we propose that episodic bursts from massive protostars are produced in groups of successive flares of different, possibly decreasing, magnitude. Some properties of the burst of S255-IR derived in Caratti o Garatti et al. (2017) are consistent with our results. Its luminosity increased by a factor 3.6-5.1 making it a 2-mag burst, which are amongst the most common bursts we predict. Its accretion rate of $(5 \pm 2) \times 10^{-3} \mathrm{M}_{\odot} \mathrm{yr}^{-1}$ fits the lower limit of accretion rates that we predict for 2 -mag bursts $\left(3 \times 10^{-3} \mathrm{M}_{\odot} \mathrm{yr}^{-1}\right)$. Note that this observed accretion rate has been estimated using a canonical value for the protostellar radius which corresponds the standard radius of a massive star of the mass of NIRS3 at the zero-age- mainsequence time. As indicated in Caratti o Garatti et al. (2017), the accretion rate would be larger if one assumes that the protostar is bloating. Indeed, if the radius is larger more mass has to be accreted to produce a similar burst energy. One should also keep in mind that our model accretion rates were derived neglecting the formation of close (spectroscopic) companions to the MYSOs, which have been shown to be consistent with the disc fragmentation and accretiondriven burst scenario in the context of massive protostars (Meyer et al. 2018). Hence, the observed accretion rate, which corresponds well to our minimal predictions, is probably a lower bound to its real value, and our predictions may be slightly overestimated.

While the observed and model burst durations remain within the same order of magnitude, a difference seems to be present nevertheless. The value of $1.5 \mathrm{yr}$ (Caratti o Garatti et al. 2017) is shorter than the lower limit of our predictions of $3.0 \mathrm{yr}$ for that kind of 2-mag bursts (our Table. 2). However, very recent results obtained by monitoring the maser emission associated with NIRS3 solves this discrepancy. The radio observations of Szymczak et al. (2018) show that the methanol maser flare of S255IR started in February 2014 already, i.e. before the estimated date of Caratti o Garatti et al. (2017). This difference is primarily due to their simplified initial analysis of light echo which neglected multiple scattering. Therefore, the estimated burst duration of $1.5 \mathrm{yr}$ represents a lower bound. The radio observations point to an effective burst length in the order of $\approx 2.0 \mathrm{yr}$ which brings the burst of S255-IR closer to our minimal prediction of $3.0 \mathrm{yr}$.

By coincidence another MYSO accretion event was detected at about the same time when NIRS3 was bursting. However, the burst from NGC6334I-MM1 could be observed at $\mathrm{mm} / \mathrm{submm}$ wavelengths only (Hunter et al. 2017). From the quadruple rise of the dust continuum emission a sustained luminosity surge by a factor of $70 \pm 20$ was derived. It suggests that the burst from NGC6334IMM1 was a major one which possibly lasts longer than that from NIRS3 which ceased after $\sim 2.0 \mathrm{yr}$. This conjecture is also supported by the duration of the flare in different maser transitions, which lasts longer than 3 years (MacLeod et al. 2018). Similar to NIRS3, this event was also accompanied by strong flares of $6.7 \mathrm{GHz}$ methanol and other maser transitions of various molecules, including new components which emerged due to the burst (Hunter et al. 2018, MacLeod et al. 2018). High resolution ALMA and VLA data revealed the presence of an outflow with a dynamic age of about $170 \mathrm{yr}$, which suggests that there was another recent accretion burst. A decrease in the water maser emission around MM1 also suggests the presence of high infrared radiation density in this region (Brogan et al.2018). The analysis of the maser data provides evidence that there were two earlier maser flares, which hints at the recurrent nature of the flares in this object (MacLeod et al. 2018).

\section{CONCLUSION}

In this study, we have numerically explored the mass accretion history of a series of massive protostars forming during the gravitational collapse of $100 \mathrm{M}_{\odot}$ pre-stellar clouds. Our threedimensional gravito-radiation-hydrodynamics simulations follow the formation and early $(60 \mathrm{kyr})$ evolution of gravitationallyunstable circumstellar discs that surround young protostars in the spirit of Meyer et al. (2017 2018). Massive fragments that form in the outer disk through gravitational fragmentation regularly generate sudden increases of the accretion rate when migrating towards the protostar and produce violent accretion-driven luminosity bursts accompanying the background variability of the protostellar lightcurves. We analyzed the characteristics, the properties and the occurrence of those bursts. All our MYSOs have accretion rate histories exhibiting a gradual increase in the accretion rate variability, from a smooth, burstless accretion phase during the initial gravitational collapse, through a variable accretion phase with $\dot{M}$ varying around the canonical value of $10^{-3} \mathrm{M}_{\odot} \mathrm{yr}^{-1}$ (Hosokawa \& Omukai 2009) in the early $10 \mathrm{kyr}$ of the disk evolution, and finally to a violent accretion phase with multiple luminous bursts. We classified the bursts in terms of their peak luminosity. More specifically, we defined 1-mag, 2-mag, 3-mag, and 4-mag luminosity bursts as those having the peak luminosity factors 2.512 , $2.512^{2} \approx 6.3,2.512^{3} \approx 15.9$, and $2.512^{4} \approx 39$ higher than the luminosity in the quiescent phase. Interestingly, the inclusion of stellar motion in the simulation slightly accelerates the fragmentation of the disc and the development of strong bursts during the early phase $(\leqslant 40 \mathrm{kyr})$ of its evolution. However, the absence/presence in the numerical setup of the effects of the stellar motion neither strongly affected nor dramatically modified the burst occurrence and the main burst characteristics.

The main prediction of this study is the scaling between burst magnitude and flare duration: the more intense (3- and 4-mag) the burst, the rarer its occurrence and the shorter $(\simeq$ yr $)$ its duration, and so is the associated observation probability. The burst occurrence increases as a function of the protostellar age, at least within the computed time limit of $60 \mathrm{kyr}$. The same is true for the peak stellar luminosity during the burst. Very luminous (3- and 4mag) outbursts are generated at later times $(\geqslant 40 \mathrm{kyr})$, i.e. they are less frequent than more fainter flares associated with moderate accretion events. Our analysis demonstrates that the most luminous bursts accrete at higher rates and over smaller timescales than fainter and longer ones. While our MYSOs exhibit about a few dozen of 1-mag bursts over their early pre-main sequence phase, only about 4-8 violent 4-mag bursts develop over the pre-main sequence phase. Their occurrence is consistent with the number of close/spectrosopic companions to the MYSOs (Mahy et al. 2013, Kobulnicky et al. 2014), with which such violent events may be simultaneously associated (Meyer et al.2018). We note that our limited number of simulations does no exhibit yet clear trends in terms of burst properties and/or occurrence as a function of the initial properties (rigidly-rotating cores with kinetic-to-gravitational energy ratios $\beta \approx 4$ to $10 \%$ ), although bursts tend to appear slightly sooner as $\beta$ increases. More numerical investigations such as a parameter study exploring the pre-stellar core internal structure and providing us with more statistics, are required to address this question. 
Although the total time our protostars spend in the burst phase represents only a small fraction $(\approx 1.7 \%)$ of their early formation phase, the mass accreted during the bursts constitutes a significant fraction (up to $50 \%$ ) of their final mass. The strongest, 4mag bursts account for $(\approx 0.1 \%$ ) of the early $60 \mathrm{kyr}$ pre-main sequence life of MYSOs. While not being frequent, we should nevertheless expect modern observation facilities to record signatures of more and more similar bursts in the years to come. Finally, we discuss our results in the light of our knowledge of observations of flares from MYSOs, i.e. the flare of the $10 \mathrm{M}_{\odot}$ massive Class I eruptive variable V723 Carinae (Tapia et al. 2006), the disk-mediated burst observed from the young massive stellar object S255IR NIRS 3 (Caratti o Garatti et al.2017) and the eruption in the massive protostellar system NGC6334I-MM1 (Hunter et al. 2017). According to our study, we suggest that the burst activity of S255IR NIRS 3 and NGC6334I-MM1 may have just started and it might further evolve as a series of flares. This conjecture is supported by the observational data hinting at a possible recurrent nature of the flares in these sources. Our study constitutes a step towards the understanding of the burst phenomenon in the high-mass star context, and further work as well as getting more observational data are necessary to accurately compare our theoretical predictions to measures and properly understand the mechanisms of bursts from young high-mass stars.

\section{ACKNOWLEDGEMENTS}

The authors thank the anonymous referee for their useful advice and suggestions which greatly improved the manuscript. D. M.A. Meyer thanks T. Hosokawa for kindly sharing his pre-main sequence stellar evolutionary tracks and for discussions on stellar motion, and W. Kley for his expertise in radiation-hydrodynamics with the PLUTO code. E. I. Vorobyov and A. M. Sobolev acknowledges support from the Russian Science Foundation grant 18-12-00193.

\section{REFERENCES}

Adams F. C., Ruden S. P., Shu F. H., 1989, ApJ, 347, 959

Banerjee R., Pudritz R. E., Anderson D. W., 2006, MNRAS, 373, 1091

Beuther H., Walsh A. J., Johnston K. G., Henning T., Kuiper R., Longmore S. N., Walmsley C. M., 2017, A\&A, 603, A10

Bitsch B., Morbidelli A., Lega E., Crida A., 2014, A\&A, 564, A135

Black D. C., Bodenheimer P., 1975, ApJ, 199, 619

Bonnell I. A., Bate M. R., 1994, MNRAS, 271

Bonnell I. A., Bate M. R., Zinnecker H., 1998, MNRAS, 298, 93

Breen S. L., Ellingsen S. P., Contreras Y., Green J. A., Caswell J. L., Stevens J. B., Dawson J. R., Voronkov M. A., 2013, MNRAS, 435, 524

Brogan C. L., Hunter T. R., Cyganowski C. J., Chibueze J. O., Friesen R. K., Hirota T., MacLeod G. C., McGuire B. A., Sobolev A. M., 2018, ApJ, 866, 87

Burns R. A., 2018, in Tarchi A., Reid M. J., Castangia P., eds, Astrophysical Masers: Unlocking the Mysteries of the Universe Vol. 336 of IAU Symposium, Water masers in bowshocks: Addressing the radiation pressure problem of massive star formation. pp 263-266

Burns R. A., Handa T., Imai H., Nagayama T., Omodaka T., Hirota T., Motogi K., van Langevelde H. J., Baan W. A., 2017, MNRAS, 467, 2367 Burns R. A., Handa T., Nagayama T., Sunada K., Omodaka T., 2016, MNRAS, 460, 283

Caratti o Garatti A., Stecklum B., Garcia Lopez R., Eisloffel J., Ray T. P., Sanna A., Cesaroni R., Walmsley C. M., Oudmaijer R. D., de Wit W. J., Moscadelli L., Greiner J., Krabbe A., Fischer C., Klein R., Ibanez J. M., 2017, Nature Physics, 13, 276
Caratti o Garatti A., Stecklum B., Linz H., Garcia Lopez R., Sanna A., 2015, A\&A, 573, A82

Cesaroni R., Galli D., Lodato G., Walmsley M., Zhang Q., 2006, Nature, 444,703

Cesaroni R., Hofner P., Araya E., Kurtz S., 2010, A\&A, 509, A50

Cesaroni R., Moscadelli L., Neri R., Sanna A., Caratti o Garatti A., Eisloffel J., Stecklum B., Ray T., Walmsley C. M., 2018, A\&A, 612, A103

Cesaroni R., Sánchez-Monge Á., Beltrán M. T., Johnston K. G., Maud

L. T., Moscadelli L., Mottram J. C., 2017, A\&A, 602, A59

Chen X., Ren Z., Zhang Q., Shen Z., Qiu K., 2017, ApJ, 835, 227

Chini R., Hoffmeister V. H., Nasseri A., Stahl O., Zinnecker H., 2012, MNRAS, 424, 1925

Commerçon B., Hennebelle P., Henning T., 2011, ApJ, 742, L9

Commerçon B., Teyssier R., Audit E., Hennebelle P., Chabrier G., 2011, A\&A, 529, A35

Cunningham N. J., Moeckel N., Bally J., 2009, ApJ, 692, 943

Flock M., Fromang S., González M., Commerçon B., 2013, A\&A, 560, A43

Forgan D. H., Ilee J. D., Cyganowski C. J., Brogan C. L., Hunter T. R., 2016, MNRAS, 463, 957

Fuente A., Neri R., Martín-Pintado J., Bachiller R., Rodríguez-Franco A., Palla F., 2001, A\&A, 366, 873

Fujisawa K., Yonekura Y., Sugiyama K., Horiuchi H., Hayashi T., Hachisuka K., Matsumoto N., Niinuma K., 2015, The Astronomer's Telegram, 8286

Gammie C. F., 1996, ApJ, 462, 725

Ginsburg A., Bally J., Goddi C., Plambeck R., Wright M., 2018, ApJ, 860, 119

Goedhart S., Gaylard M. J., van der Walt D. J., 2003, MNRAS, 339, L33

Haemmerlé L., Eggenberger P., Meynet G., Maeder A., Charbonnel C., 2016, A\&A, 585, A65

Haemmerlé L., Eggenberger P., Meynet G., Maeder A., Charbonnel C., Klessen R. S., 2017, A\&A, 602, A17

Haemmerlé L., Peters T., 2016, MNRAS, 458, 3299

Harries T. J., 2015, MNRAS, 448, 3156

Harries T. J., Douglas T. A., Ali A., 2017, MNRAS, 471, 4111

Hirano S., Hosokawa T., Yoshida N., Kuiper R., 2017, Science, 357, 1375

Hosokawa T., Hirano S., Kuiper R., Yorke H. W., Omukai K., Yoshida N., 2016, ApJ, 824, 119

Hosokawa T., Omukai K., 2009, ApJ, 691, 823

Hunter T. R., Brogan C. L., MacLeod G., Cyganowski C. J., Chandler C. J., Chibueze J. O., Friesen R., Indebetouw R., Thesner C., Young K. H., 2017, ApJ, 837, L29

Hunter T. R., Brogan C. L., MacLeod G. C., Cyganowski C. J., Chibueze J. O., Friesen R., Hirota T., Smits D. P., Chandler C. J., Indebetouw R., 2018, ApJ, 854, 170

Ilee J. D., Cyganowski C. J., Nazari P., Hunter T. R., Brogan C. L., Forgan D. H., Zhang Q., 2016, MNRAS, 462, 4386

Johnston K. G., Robitaille T. P., Beuther H., Linz H., Boley P., Kuiper R., Keto E., Hoare M. G., van Boekel R., 2015, ApJ, 813, L19

Keto E., Wood K., 2006, ApJ, 637, 850

Klassen M., Pudritz R. E., Kuiper R., Peters T., Banerjee R., 2016, ApJ, 823,28

Kobulnicky H. A., Kiminki D. C., Lundquist M. J., Burke J., Chapman J., Keller E., Lester K., Rolen E. K., Topel E., Bhattacharjee A., Smullen R. A., Vargas Álvarez C. A., Runnoe J. C., Dale D. A., Brotherton M. M., 2014, ApJS, 213, 34

Kolb S. M., Stute M., Kley W., Mignone A., 2013, A\&A, 559, A80

Kratter K. M., Matzner C. D., Krumholz M. R., Klein R. I., 2010, ApJ, 708,1585

Kraus S., Kluska J., Kreplin A., Bate M., Harries T. J., Hofmann K.-H., Hone E., Monnier J. D., Weigelt G., Anugu A., de Wit W. J., Wittkowski M., 2017, ApJ, 835, L5

Krumholz M. R., Klein R. I., McKee C. F., 2007, ApJ, 656, 959

Langer N., 2012, ARA\&A, 50, 107

Lodato G., Clarke C. J., 2011, MNRAS, 413, 2735

Machida M. N., Matsumoto T., 2011, MNRAS, 413, 2767 
MacLeod G. C., Smits D. P., Goedhart S., Hunter T. R., Brogan C. L., Chibueze J. O., van den Heever S. P., Thesner C. J., Banda P. J., Paulsen J. D., 2018, MNRAS, 478, 1077

Mahy L., Rauw G., De Becker M., Eenens P., Flores C. A., 2013, A\&A, 550, A27

Matsumoto T., Machida M. N., Inutsuka S.-i., 2017, ApJ, 839, 69

Maud L. T., Hoare M. G., Galván-Madrid R., Zhang Q., de Wit W. J., Keto

E., Johnston K. G., Pineda J. E., 2017, MNRAS, 467, L120

Meru F., Bate M. R., 2010, MNRAS, 406, 2279

Meyer D. M.-A., Kuiper R., Kley W., Johnston K. G., Vorobyov E., 2018, MNRAS, 473, 3615

Meyer D. M.-A., Langer N., Mackey J., Velázquez P. F., Gusdorf A., 2015, MNRAS, 450, 3080

Meyer D. M.-A., Mackey J., Langer N., Gvaramadze V. V., Mignone A., Izzard R. G., Kaper L., 2014, MNRAS, 444, 2754

Meyer D. M.-A., Vorobyov E. I., Kuiper R., Kley W., 2017, MNRAS, 464, L90

Mignone A., Bodo G., Massaglia S., Matsakos T., Tesileanu O., Zanni C., Ferrari A., 2007, ApJS, 170, 228

Mignone A., Zanni C., Tzeferacos P., van Straalen B., Colella P., Bodo G., 2012, ApJS, 198, 7

Moscadelli L., Sanna A., Goddi C., Walmsley M. C., Cesaroni R., Caratti o Garatti A., Stecklum B., Menten K. M., Kraus A., 2017, A\&A, 600, L8

Nayakshin S., Lodato G., 2012, MNRAS, 426, 70

Peters T., Banerjee R., Klessen R. S., Mac Low M.-M., Galván-Madrid R., Keto E. R., 2010, ApJ, 711, 1017

Purser S. J. D., Lumsden S. L., Hoare M. G., Cunningham N., 2018, MNRAS, 475, 2

Purser S. J. D., Lumsden S. L., Hoare M. G., Urquhart J. S., Cunningham N., Purcell C. R., Brooks K. J., Garay G., Gúzman A. E., Voronkov M. A., 2016, MNRAS, 460, 1039

Regály Z., Vorobyov E., 2017, A\&A, 601, A24

Reiter M., Kiminki M. M., Smith N., Bally J., 2017, MNRAS, 470, 4671

Rice W. K. M., Lodato G., Armitage P. J., 2005, MNRAS, 364, L56

Richling S., Yorke H. W., 1997, A\&A, 327, 317

Rogers P. D., Wadsley J., 2012, MNRAS, 423, 1896

Rosen A. L., Krumholz M. R., McKee C. F., Klein R. I., 2016, MNRAS, 463, 2553

Rosen A. L., Krumholz M. R., Oishi J. S., Lee A. T., Klein R. I., 2017, Journal of Computational Physics, 330, 924

Samal M. R., Chen W. P., Takami M., Jose J., Froebrich D., 2018, MNRAS, 477, 4577

Sana H., de Mink S. E., de Koter A., Langer N., Evans C. J., Gieles M., Gosset E., Izzard R. G., Le Bouquin J.-B., Schneider F. R. N., 2012, Science, 337, 444

Schneider F. R. N., Izzard R. G., Langer N., de Mink S. E., 2015, ApJ, 805,20

Scholz A., Froebrich D., Wood K., 2013, MNRAS, 430, 2910

Seifried D., Banerjee R., Klessen R. S., Duffin D., Pudritz R. E., 2011, MNRAS, 417, 1054

Sobolev A. M., Cragg D. M., Godfrey P. D., 1997, A\&A, 324, 211

Stecklum B., Caratti o Garatti A., Cardenas M. C., Greiner J., Kruehler T., Klose S., Eisloeffel J., 2016, The Astronomer's Telegram, 8732

Stecklum B., Caratti o Garatti A., Hodapp K., Linz H., Moscadelli L., Sanna A., 2018, in Tarchi A., Reid M. J., Castangia P., eds, Astrophysical Masers: Unlocking the Mysteries of the Universe Vol. 336 of IAU Symposium, Infrared variability, maser activity, and accretion of massive young stellar objects. pp 37-40

Stecklum B., Heese S., Wolf S., Garatti A. C. o., Ibanez J. M., Linz H., 2017, ArXiv e-prints

Szymczak M., Olech M., Sarniak R., Wolak P., Bartkiewicz A., 2018, MNRAS, 474, 219

Szymczak M., Olech M., Wolak P., Gérard E., Bartkiewicz A., 2018, A\&A, 617, A80

Tanaka K. E. I., Tan J. C., Zhang Y., 2017, ApJ, 835, 32

Tapia M., Persi P., Bohigas J., Roth M., Gómez M., 2006, MNRAS, 367, 513
Tapia M., Roth M., Persi P., 2015a, MNRAS, 448, 1402

Tapia M., Roth M., Persi P., 2015b, MNRAS, 446, 4088

Testi L., 2003, in De Buizer J. M., van der Bliek N. S., eds, Galactic Star Formation Across the Stellar Mass Spectrum Vol. 287 of Astronomical Society of the Pacific Conference Series, Intermediate Mass Stars (Invited Review). pp 163-173

Truelove J. K., Klein R. I., McKee C. F., Holliman II J. H., Howell L. H., Greenough J. A., Woods D. T., 1998, ApJ, 495, 821

Vaidya B., Fendt C., Beuther H., Porth O., 2011, ApJ, 742, 56

Vorobyov E. I., 2010, ApJ, 713, 1059

Vorobyov E. I., Basu S., 2005, ApJ, 633, L137

Vorobyov E. I., Basu S., 2015, ApJ, 805, 115

Vorobyov E. I., Elbakyan V., Hosokawa T., Sakurai Y., Guedel M., Yorke H., 2017, A\&A, 605, A77

Vorobyov E. I., Elbakyan V. G., 2018, A\&A, 618, A7

Vorobyov E. I., Elbakyan V. G., Plunkett A. L., Dunham M. M., Audard M., Guedel M., Dionatos O., 2018, A\&A, 613, A18

Wang Y., Beuther H., Bik A., Vasyunina T., Jiang Z., Puga E., Linz H., Rodón J. A., Henning T., Tamura M., 2011, A\&A, 527, A32

Yorke H. W., Sonnhalter C., 2002, ApJ, 569, 846

Zhao B., Caselli P., Li Z.-Y., Krasnopolsky R., 2018, MNRAS, 473, 4868

Zinchenko I., Liu S.-Y., Su Y.-N., Salii S. V., Sobolev A. M., Zemlyanukha P., Beuther H., Ojha D. K., Samal M. R., Wang Y., 2015, ApJ, 810, 10 Zinchenko I., Liu S.-Y., Su Y.-N., Sobolev A. M., 2017, A\&A, 606, L6 Zinchenko I., Liu S.-Y., Su Y.-N., Zemlyanukha P., 2018, in Cunningham M., Millar T., Aikawa Y., eds, IAU Symposium Vol. 332 of IAU Symposium, Multiline observations of S255IR with ALMA. pp 270-273 